\title{
miR-4293 Upregulates IncRNA WFDC21P by Directly Suppressing mRNA-Decapping Enzyme 2 to Promote Lung Carcinoma Proliferation
}

\section{Qian Zhang}

Binzhou Medical University

\section{Yun-Fei Yan}

Binzhou Medical University https://orcid.org/0000-0001-5273-5314

Qing Lv

Binzhou Medical University

You-Jie Li

Binzhou Medical University

\section{Ran-Ran Wang}

Binzhou Medical University

\section{Guang-Bin Sun}

Binzhou Medical University

Li Pan

Binzhou Medical University

Jin-Xia Hu

Binzhou Medical University

Ning Xie

Yantaishan Hospital

Can Zhang

Massachusetts General Hospital

Bao-Cheng Tian

Binzhou Medical University

Fei Jiao

Binzhou Medical University

Sen Xu

Binzhou Medical University

\section{Ping-Yu Wang}

Binzhou Medical University

Shu-Yang Xie ( $\square$ shuyangxie@163.com )

Binzhou Medical University 
Research

Keywords: WFDC21P, miR-4293, lung carcinoma, DCP2, cell proliferation

Posted Date: August 20th, 2020

DOl: https://doi.org/10.21203/rs.3.rs-60831/v1

License: (c) (i) This work is licensed under a Creative Commons Attribution 4.0 International License. Read Full License

Version of Record: A version of this preprint was published at Cell Death \& Disease on July 23rd, 2021. See the published version at https://doi.org/10.1038/s41419-021-04021-y. 


\section{Abstract}

Background: Emerging evidence shows that IncRNA WFDC21P could promote STAT3 phosphorylation and microRNA 4293 SNP is associated with the risk of carcinomas, but the oncogenic functions of WFDC21P and miR-4293 in lung carcinoma are unclear.

Methods: mRNA sequencing of lung carcinoma and control para-carcinoma tissues was performed to screen the potential targets. WFDC21P and miR-4293 levels were evaluated in lung carcinoma cells and tissues by qRT-PCR. The function of WFDC21P and miR-4293 on proliferation, apoptosis and metastasis were assessed by MTT, FACS, western blot, transwell assays, colony formation assays and xenografts experiment. RNA immunoprecipitation assays were implemented to verify the relationship between WFDC21P and mRNA-decapping enzyme 2 (DCP2). Furthermore, gain/loss of miR-4293 functions were used to determine its targeting relationship of DCP2.

Results: WFDC21P expression is markedly enhanced in lung carcinoma tissue and cells. Moreover, WFDC21P promotes tumor cell proliferation and metastasis but suppresses apoptosis. Mechanistic investigations identified DCP2 can directly bind to WFDC21P and down-regulates its expression. DCP2 as a direct target of miR-4293 and its expression is suppressed by miR-4293. Consequently, miR-4293 can further promote WFDC21P expression by regulating DCP2. With positive correlation to WFDC21P expression, miR-4293 also plays oncogenic role in lung carcinoma. Furthermore, knockdown of WFDC21P results in functional attenuation of miR-4293 on tumor promotion. In vivoxenograft growth is also promoted by both WFDC21P and miR-4293.

Conclusion: Our results establish oncogenic roles for both WFDC21P and miR-4293, and demonstrate that interactions between miRNAs and IncRNAs through DCP2 are important in lung carcinoma pathogenesis.

\section{Background}

Lung carcinoma remains a leading cause of cancer death globally. In spite of advances in early detection and standard treatment, lung cancer is often diagnosed at an advanced stage and has a poor prognosis. $[1,2]$ The first step in developing effective diagnosis and therapy is to elucidate the molecular mechanism operative in lung carcinoma. Recent large-scale genomic analyses of lung carcinoma identified many potential targets that can be exploited in diagnosis and therapies. $[3,4]$

Non-coding RNAs (ncRNAs), such as long non-coding RNAs (IncRNAs) and microRNAs (miRNAs), are regulators of intracellular and intercellular signaling in lung cancer and modulate cell signaling to control diverse cellular processes, including proliferation, migration and apoptosis. Compared to miRNAs, IncRNAs exhibit more wide range of size and functions. [5] In tumorigenesis of lung carcinoma, some IncRNAs serve as tumor suppressive roles such as MEG3 and GAS5, while some are oncogenes like HOTAIR and MALAT1.[6-9] As important regulators in gene expression network, IncRNA can modulate 
mRNA stability, translation and post-translation in cytoplasm. In nucleus, IncRNAs are capable of controlling nuclear architecture and transcription.[10]

Among the versatile functions of IncRNA, the crosstalk between IncRNA and miRNA is probably the most reported. As the competing endogenous RNA, IncRNAs can act as miRNA decoys to modulate gene expression.[11-13] Indeed, miRNAs can also directly or indirectly affect the expression of IncRNAs.[14] In the process of miRNA-mediated gene silencing, miRNA-induced silencing complexes can recruit the cellular mRNA decay machinery to degrade mRNAs and IncRNAs in which decapping complexes are important factors. $[15,16]$ Components of decapping complexes, including DCP1 and DCP2, can remove 7-methylguanosine (m7G) cap to initiate mRNAs decay. [17-19] Similarly, decapping complexes also modulate the expression of IncRNAs through RNA degradation, which serves a vital role in transcriptional regulation specifically at inducible genes.[20] The role of IncRNA degradation in the pathogenesis of carcinoma has not been clarified.

WFDC21P is a pseudogene, belonging to WAP four-disulfide core domain family which has been reported playing important roles in pathogenesis of lung carcinoma.[21, 22] Moreover, WFDC21P was identified as a positive regulator of dendritic cell differentiation by promoting STAT3 phosphorylation.[23] However, the function of WFDC21P in lung carcinoma is still unknown. In this study, we presented evidence that WFDC21P could be degraded by directly binding to DCP2, and that WFDC21P is highly expressed in lung carcinoma tissue and promotes cell proliferation and migration, but inhibits apoptosis. More importantly, we revealed that miR-4293 can elevate the expression of WFDC21P by regulating DCP2. Here, we demonstrate that miR-4293 plays a proto-oncogenic role in lung carcinoma. In addition, WFDC21P and miR-4293 are found possibly participating in a common pathway to regulate STAT3 activation. The oncogenic potential of miR-4293 can be attenuated by blocking WFDC21P. What's more, both WFDC21P and miR-4293 enhance xenograft growth in vivo.

\section{Materials And Methods}

\section{Lung Carcinoma Tissues}

Sectioned lung tissues were collected between August 1, 2016 and July 31, 2018 from the Inpatient Department of Medical Oncology, Yantai Shan Hospital, the Teaching Hospital of Binzhou Medical University (Yantai, China). The experiments were performed in accordance with the relevant guidelines of the Code of Ethics of the World Medical Association for experiments involving humans and the Medical Ethics Committee of Binzhou Medical University. Patients provided written informed consent and the study procedures were fully explained before study inclusion. Nineteen patients (10 males and 9 females, aged 31-74 years) who were pathologically diagnosed with NSCLC for the first time and had not yet received chemotherapy, were included in the present study. Fresh NSCLC tissues and controls were obtained from the patients who underwent surgery.

\section{mRNA transcriptome library preparation and sequencing}


Total RNAs from 3 NSCLC and control para-carcinoma tissues were isolated. The preparation of mRNA transcriptome libraries and deep sequencing were performed by Novogene Bioinformatics Technology Cooperation (Beijing, China). mRNA was gathered and strand-specific sequencing libraries were generated following manufacture's recommendations. RNA-Seq was performed on an Illumina Hiseq 2000 platform and 100 bp paired-end reads were generated according to Illumina's protocol.

\section{RNA-Seq data analysis}

The adapter sequences were removed from the raw sequencing data and the individual libraries were converted to the FASTQ format. Sequence reads were aligned to the human genome (hg19) with TopHat2 and the resulting alignment files were reconstructed by Cufflinks50 and Scripture51. For mRNA analyses, the RefSeq database (Build 37.3) was chosen as the annotation references.

The read counts of each transcript were normalized to the length of the individual transcript and to the total mapped fragment counts in each sample and expressed as fragments per kilo-base of exon per million fragments mapped (FPKM) in each sample. The mRNA differential expression analyses for carcinoma versus para-carcinoma (CA vs PARA). An adjusted $P$ value $<0.05$ (Student's $t$-test with Benjamini-Hochberg false discovery rate (FDR) adjustment) was used as the cut-off for significantly differentially expressed genes. Differentially expressed genes were analyzed by enrichment analyses to detect over-represented functional terms present in the genomic background.

\section{Real-time qRT-PCR}

Real-time qRT-PCR was performed as previously described [24, 25]. Primers used to amplify miR-4293 were: forward: 5'-AUAUGUUGGUACUCCCAUUTT-3'; reverse: 5'- AAUGGGAGUACCAACAUAUTT- 3'. Human $5 S$ rRNA served as positive control. The primers used to amplify STAT3 were: forward: 5 '-

TGCGGAGAAGCATCGTGAGT-3'; reverse: 5'- CCTCCAATGCAGGCAATCTGT-3'. The primers used to amplify WFDC21P were forward: 5'-CCAAGACCTGAGCCCTGTAA-3'; reverse: 5'-ATAGAGGTGGCTGTCTGATGCT 3' (Supplemental Table 1). qRT-PCR was performed on a Rotor Gene RG-3000 system (Qiagen, Germany) under the following reaction condition: initial denaturation at $95^{\circ} \mathrm{C}$ for $30 \mathrm{~s}$, followed by 40 cycles at $95^{\circ} \mathrm{C}$ for $5 \mathrm{~s}$, annealing at $60^{\circ} \mathrm{C}$ for $20 \mathrm{~s}$, and extension at $72{ }^{\circ} \mathrm{C}$ for $30 \mathrm{~s}$. GAPDH cDNAs served as positive control.

\section{Cell Lines}

The human cell lines (A549, H1299, H1975 and HBE) were obtained from the Shanghai Institute of Cell Biology, China. Cells were cultured in RPMI-1640 medium (Gibco, Grand Island, New York, USA) supplemented with $10 \%$ fetal bovine serum (Gibco) in a standard humidified incubator with $5 \% \mathrm{CO}_{2}$ at $37^{\circ} \mathrm{C}$.

\section{Cell Transfection}

Cells were seeded in 96-well plates or 6-well plates. Transfection was performed in triplicate at approximately 60\% confluence using Lipofectamine TM 2000 (Invitrogen, Carlsbad, CA, USA) according 
to the manufacturer's instructions.

\section{MTT Assay}

Cells proliferation was measured with an MTT assay as our previous reports [26, 27]. Cells $\left(1 \times 10^{4}\right)$ in each well of a 96-well flat bottom microtiter plates were treated with microRNAs (Supplemental Table 2) or plasmids for $48 \mathrm{~h}$. Four hours before the end of incubation, $10 \mu \mathrm{L}$ MTT (Sigma, St Louis, MO, USA, $5 \mathrm{mg} / \mathrm{mL}$ ) was added to each well. Supernatant was then removed and $100 \mu \mathrm{L}$ DMSO (Sigma) was added and the optical density (OD) was measured $(570 \mathrm{~nm})$ using an ELISA reader (Multiskan FC, Thermo Fisher Scientific, Boston, MA, USA).

\section{Apoptosis Assays}

Apoptotic cells were detected using an Annexin V-FITC/PI kit (KeyGEN Biotech. Co. Ltd., Nanjing, China) according to the manufacturer's instructions. A total of $1 \times 10^{4}$ cells was collected and analyzed by flow cytometry as previous described [24, 25].

\section{Western Blot}

Western blot analysis was performed as described previously. The antibodies used were as follows: rabbit anti-human STAT3 (1:500; Bioss, Ltd), rabbit antihuman p-STAT3 (1:800), mouse anti-human BIRC3 (1:500), rabbit anti-human Bax (1:500), Bcl2 (1:500), rabbit anti-human Caspase 3 (1:1000;), rabbit anti-human Caspase 8(1:1000), rabbit anti-human Caspase 9 (1:1000), and rabbit anti-human GAPDH (1:3000) all from Bioworld Technology, Ltd).

\section{Transwell Migration Assay}

Transwell migration assays were performed using Corning Costar Transwell chambers with $8 \mu \mathrm{m}$ pore size membranes (Sigma, St Louis, MO, USA). After transfection, cancer cells incubated in $500 \mu \mathrm{L}$ serum free medium were seeded into the upper chamber. The lower chamber was filled with $600 \mu \mathrm{L} 1640$ medium supplemented with $10 \%$ calf serum. After 24 hours, cells on the upper surface of the membrane were removed with cotton swabs. Lower chamber cells were stained with $1 \%$ crystal violet (Sigma) in $2 \%$ ethanol for 20 min. Excess crystal violet was removed by quickly immersing the insert in $\mathrm{dd} \mathrm{H}_{2} \mathrm{O}$ for 3-4 s. Stained cells were counted under a microscope (DM6000B, Leica). The assay was repeated three times for each group.

\section{Colony Formation}

After transfection in 6 wells plates, cancer cells were incubated for 48 hours. Then, transfected cells were harvested, and $1.5 \times 10^{3}$ cells per treatment group were seeded into $6 \mathrm{~cm}$ plates for colony formation. On the 12 th day, colonies were stained with $0.25 \%$ crystal violet. Colonies were countered and reported as the number \pm SEM.

\section{RNA Immunoprecipitation}


RNA immunoprecipitation (RIP) experiments were performed using a Magna RIP RNA-Binding Protein Immunoprecipitation Kit (Merk millipore, Darmstadt, Germany) according to the manufacturer's instructions. Antibodies for RIP assays against DCP2 were purchased from Abcam-Trading Company (Shanghai, China).

\section{Continuously Monitor Cell Migration}

To continuously monitor cancer cell migration, $1 \times 10^{4}$ cells per well were seeded into the top chamber of a CIM plate, which was then incubated on the RTCA station (xCELLigence System, Roche, Mannheim, Germany). Changes in cell migration were monitored in real-time.

\section{Xenografts in Mice}

A549 cells stably expressed WFDC21P, or untreated A549 cells $\left(5 \times 10^{6}\right)$ in $0.1 \mathrm{~mL}$ PBS, transfected with miR-4293, WFDC21P-small interfering RNAs (siRNAs) (Supplemental Table 2), WFDC21P were transplanted subcutaneously into the right or left flanks of 5-6-week-old male BALB/c-nu/nu mice weighing 18-20 g (Charles River. Beijing, China). The maximum and minimum tumor diameters were measured every $3 \mathrm{~d}$. The second time of injection the xenografts with siRNA or miRNA (Supplemental Table 2) was performed at the 14 day after first time treatment. The mice were house in specific-pathogen free (SPF) environment. Tumor volume $\left(\mathrm{mm}^{3}\right)=A \times B^{2} / 2$, where $A$ and $B$ are the maximum and minimum tumor diameters, respectively. After 4 weeks, nu mice were given euthanasia by amobarbital injection of 3 times standard does. The tumors were anatomized and weighed. All animal experiments were approved by the Committee on the Ethics of Animal Experiments of Binzhou Medical University and performed in accordance with the National Institutes of Health guide for the care and use of Laboratory animals.

\section{Statistics}

SPSS 22.0 software (IBM Corp., Armonk, NY, USA) was used to analyze statistical significance. Normally distributed data were expressed as mean \pm SD, and Student's $t$ test was used to compare two averages and ANOVA was used for mean comparison of multiple groups. If the assumption of homogeneity of variance is accepted or rejected, LSD test or Games-Howell test is used to compare the mean of different samples. Abnormally distributed data were expressed as median (interquartile range), the Mann-Whitney $\mathrm{U}$ test was used to compare two groups, and Kruskal-Wallis $\mathrm{H}$ test were used to compare multiple groups. Pearson's correlation was used to analyze the association between different variables. $p<0.05$ is considered statistically significant differences.

\section{Results}

\section{WFDC21P Increases in Lung Carcinoma Cells and Tissues}

Carcinoma tissue lysates prepared from 3 NSCLC patients and controls from their corresponding paracarcinoma tissues were subjected to RNA-seq analysis to detect mRNA expression. Mining of the RNAseq dataset identified that the expression of 1392 mRNAs were elevated, and 1303 were down-regulated 
(fold change $>2.0, p<0.05$ ) (Fig. 1A). Accumulated evidence indicated that WFDC family played great roles in the progression of carcinoma.[28-31] Additionally, some of them has been identified as biomarkers of diagnosis of lung cancer.[32-34] Here, the members of WFDC family were selected to do further comprehensive analysis of their roles in lung cancer. Our results demonstrated that WFDC1 was attenuated while WFDC2 was significantly elevated in carcinoma tissue. As a member of WFDC family, we also found that IncRNA-WFDC21P may have enhanced expression in carcinoma tissue (Fig. 1B). Moreover, the role of WFDC21P in tumorigenesis of lung cancer is unclear till now. Therefore, we collected more NSCLC sample tissues and corresponding non-tumor tissues to further investigate the WFDC21P expression in lung cancer. Compared with lung para-carcinoma samples, WFDC21P levels were elevated in lung carcinoma samples (Fig. 1C). Additionally, we also found that the expression of WFDC21P in cancer cell lines (A549, H1299 and H1975) was markedly elevated compared with human bronchial epithelial cells (HBE) (Fig. 1D). These results indicate that WFDC21P may play significant roles in lung carcinoma pathogenesis.

\section{WFDC21P Enhances Cell Proliferation and Migration}

In order to examine the effects of WFDC21P on cell proliferation, migration and apoptosis, overexpression or interference of WFDC21P was performed in A549 or H1975 cells (Fig. 2A and C). It was found that WFDC21P overexpression markedly enhanced A549 or H1975 cell growth (Fig. 2B and S1A), while transfection with an effective si-WFDC21P inhibited cell proliferation (Fig. 2D and S1B). The role of WFDC21P in apoptosis was also examined, and WFDC21P knockdown increased apoptosis (Fig. 2E and S1C). WFDC21P can promote STAT3 phosphorylation in dendritic cells.[23] To further investigate whether the role WFDC21P in cell apoptosis is related to STAT3 signals, the expression of apoptotic factors related to STAT3 was examined. The expression of STAT3 and anti-apoptosis factors (Bcl2 and BIRC3) was significantly attenuated in si-WFDC21P-treated cells, but was elevated by WFDC21P overexpression. WFDC21P knockdown resulted in Bax elevation, while WFDC21P overexpression decreased Bax levels. Additionally, WFDC21P knockdown resulted in higher levels of cleaved caspases (caspase-3, 8 and 9). As expected, WFDC21P overexpression suppressed cleavage of caspases. The effects of WFDC21P on the examined apoptosis factors in A549 or $\mathrm{H} 1975$ cells may be owned to the enhanced phosphorylation of STAT3 resulted from WFDC21P elevation (Fig. 2F and S1D). Therefore, we next examined the effects of WFDC21P on tumor cell migration. WFDC21P overexpression increased the number of cells penetrating the Transwell membrane. Conversely, WFDC21P knockdown attenuated the cell penetration (Fig. 2G-J and S1E-H). In the colony formation assay, overexpression of WFDC21P resulted in an elevation of colony number. As expected, WFDC21P knockdown decreased the number of colonies (Fig. 2K-N and S1I-L). These results indicate that WFDC21P is proto-oncogene in lung carcinoma.

\section{WFDC21P Interacts with, and is Regulated by DCP2}

Although WFDC21P has the function of promoting carcinoma, the detail mechanisms were still unknown. DCP2, a key component for degrading RNA, is involved in the pathogenesis of some carcinoma.[35] We assumed that WFDC21P is regulated by DCP2, and is involved in the pathogenesis of lung cancer. To investigate this hypothesis, we first screened for an effective siRNA against DCP2 (Fig. 3A). Then, we 
detected the role of DCP2 in regulating WFDC21P expression. As expected, pcDNA-WFDC21P transfection elevated WFDC21P levels compared with the scrambled control. After DCP2 knockdown, irrespective of whether WFDC21P was overexpressed or not, WFDC21P was significantly elevated compared with the scrambled control. More importantly, DCP2 knockdown combined with pcDNA-WFDC21P transfection resulted in further increased WFDC21P expression compared with control treatment (pcDNA-WFDC21P + Scram) (Fig. 3B). As a regulated target of WFDC21P, STAT3 phosphorylation was also used to evaluate the effects of DCP2 on WFDC21P. As expected, WFDC21P overexpression after attenuating DCP2 by siRNA could further enhance STAT3 phosphorylation compared with scrambled control treatment. STAT3 phosphorylation was also higher in cells co-transfected with si-DCP2 and pcDNA-WFDC21P compared with control treatment (Fig. 3C). DCP2 can bind to RNA and promote its degradation by decapping. Therefore, RNA immunoprecipitation (RIP) was performed to check the direct interaction between WFDC21P and DCP2. As a result, WFDC21P was significantly enriched by DCP2 immunoprecipitation compared with control IgG precipitation, indicating that WFDC21P directly interacts with DCP2 (Fig. 3D). These results indicate that DCP2 may promote WFDC21P degradation by directly interacting with WFDC21P.

\section{DCP2 is a Direct Target of miR-4293}

Furthermore, we predicted that DCP2 mRNA might be a target of miR-4293 by using miRNA TargetScan (http://www.targetscan.org/vert_72/) (Fig. 3E). A GFP reporter plasmid containing DCP2-3'UTR was then constructed and used to co-transfect cells with miR-4293. miR-4293 transfection resulted in considerable attenuation of both GFP intensity and the percentage of GFP-positive cells. Cells were then co-transfected with mu-4293 (containing a mutated site of DCP2 mRNA binding) and the DCP2-3'-UTR GFP reporter plasmid. mu-4293 could not reduce the percentage of GFP positive cells compared with the wide type miR-4293. To further confirm that miR-4293 binds to the DCP2-3'UTR sequence, we constructed a muDCP2-3'UTR GFP reporter plasmid (with a mutant binding site for miR-4293) in which we deleted the "CTG" sequence from the DCP2-3'UTR GFP reporter plasmid. As a result, cells, co-transfected with muDCP2-3'UTR and miR-4293, had no significant attenuation in the percentage of GFP-positive cells (Figure S2). Next, we transfected cancer cells (A549 and H1975) with miR-4293 and its inhibitor (ASO-4293). As expected, miR-4293 transfection significantly attenuated DCP2 expression compared to transfection of both ASO-4293 and scrambled control both in A549 and H1299 cells (Fig. 3F). These results indicate that miR-4293 can directly bind to the DCP2-3'UTR to regulate the expression of DCP2.

\section{miR-4293 Increases WFDC21P and STAT3 Levels through Regulating DCP2}

The above results indicate that WFDC21P is degraded by interaction with DCP2 and DCP2 expression is suppressed by miR-4293. However, the effects of miR-4293 on WFDC21P were still to be elucidated. Firstly, we prepared miR-4293 (mimic), ASO-4293 (inhibitor) and mu-4293 (mutant). A549 cells were then transfected with miR-4293, ASO-4293 or mu-4293 respectively. After transfection, miR-4293 mimic could significantly elevate miR-4293 level, which could be markedly attenuated by ASO-4293 (Fig. 3G). By detecting WFDC21P level, we found that miR-4293 treatment could markedly enhance WFDC21P 
expression compared with the scrambled control. In contrast, ASO-4293 treatment suppressed WFDC21P expression compared with the scrambled control (Fig. 3H). In addition, miR-4293 treatment further increased WFDC21P levels (Fig. 3I). These results indicate that miR-4293 elevation can promote the expression of WFDC21P.

Next, STAT3 activation was used to evaluate the relationship between WFDC21P and miR-4293. As expected, overexpression of both WFDC21P and miR-4293 resulted in more STAT3 phosphorylation compared with only WFDC21P or miR-4293 treatment (Fig. 3J). To further investigate the role of WFDC21P in stimulating STAT3 pathway, we treated WFDC21P-overexpressed cells with AG490 (a specific inhibitor to STAT3 signaling, $50 \mu \mathrm{mol} / \mathrm{L}$ ), and found that AG490 treatment reduced WFDC21Pinduced STAT3 activation (Fig. 3K).

We also found that WFDC21P, by regulating STAT3 pathway, may have a positive feedback effect on the expression of miR-4293. STAT3 pathway was deactivated by AG490 treatment, which also could result in the decreased levels of miR-4293. Moreover, miR-4293 elevation resulting from WFDC21P overexpression was further alleviated by using AG490 to deactivate STAT3 pathway (Fig. 3L). These results indicate that there might be a feedback loop between miR-4293 and WFDC21P in which miR-4293 increases WFDC21P levels and WFDC21P might upregulate miR-4293 expression by activation of STAT3 pathways.

\section{MiR-4293 and STAT3 Levels also Increased in NSCLC Cells and Tissues}

The above results indicate that WFDC21P expression and STAT3 activation can be upregulated by miR4293. However, the role of miR-4293 and STAT3 in the pathogenesis of NSCLC requires clarification. Firstly, we examined the expression of miR-4293 in carcinoma cell lines (A549, H1299 and H1975) and found that miR-4293 expression was significantly elevated compared to HBE (Fig. 4A). As expected, miR4293 elevation was also found in NSCLC tissues compared with para-carcinoma tissues (Fig. 4B). As a direct target of WFDC21P, STAT3 phosphorylation was increased in lung carcinoma cells (Fig. 4C). Similarly, STAT3 expression was also increased in lung carcinoma tissue (Fig. 4D). Based on the above results, we further analyzed possible correlations among WFDC21P, STAT3 and miR-4293 in the NSCLC samples. We found that WFDC21P expression was positively correlated with STAT3 expression $(p<0.01$, Fig. 4E). miR-4293 and WFDC21P expression was also in positive correlation ( $p=0.015$, Fig. 4F), as was the relationship between miR-4293 and STAT3 expression ( $p<0.01$, Fig. 4G).

\section{MiR-4293 Promotes Cell Proliferation and Migration}

In order to further study the role of miR-4293 in lung carcinoma, MTT assays were performed and showed that miR-4293 transfection could significantly promote A549 or H1975 cell proliferation compared with cells transfected with scrambled control or ASO-4293 inhibitor. ASO-4293 transfection suppressed cancer cell proliferation compared with the scrambled control (Fig. 5A and S3A). The apoptosis assay showed that miR-4293 treatment decreased lung cancer cell (A549 or H1975) apoptosis compared with the 
scrambled control. More importantly, miR-4293 treatment significantly increased survival capacity compared with ASO-4293 treatment (Fig. 5B and S3B).

Next, we investigated the effects of miR-4293 on apoptosis related factors. We found that the expression of anti-apoptosis factors (Bcl2 and BIRC3) could be elevated by miR-4293 transfection, but was attenuated by ASO-4293 treatment compared with scrambled control. However, the expression of Bax, a pro-apoptosis factor was suppressed by miR-4293 treatment and enhanced by ASO-4293 transfection. We also examined the expression of caspases influenced by miR-4293 and ASO-4293. miR-4293 treatment resulted in less cleavage of caspase-3, caspase-8 and caspase-9, which play essential roles in apoptosis. Whereas, ASO-4293 treatment induced the cleavage of caspase- 3 , caspase- 8 and caspase- 9 compared with miR-4293. Importantly, the apoptotic factors examined are closely related to STAT3 signaling pathways; therefore, we detected STAT3 expression and found that miR-4293 treatment could significantly enhance STAT3 phosphorylation compared with scrambled control, in contrast, ASO-4293 treatment suppressed STAT3 phosphorylation compared with miR-4293 transfection (Fig. 5C and S3C). To investigate the possible role of miR-4293 in tumor cell migration, we conducted Transwell migration assays, which demonstrated that A549 or $\mathrm{H} 1975$ cells transfected with miR-4293 have enhanced migration capacity compared with cells transfected with scrambled control (Fig. 5D and E, S3D and E). Additionally, miR-4293 treatment could significantly increase survival of A549 or H1975 cells seeded sparsely in a clonogenic assay compared with the scrambled control. Conversely, cells transfected with ASO-4293 formed fewer colonies compared with scrambled control (Fig. 5F and G, S3F and G). miR-4293 promoted cell proliferation and migration but inhibited apoptosis. These results indicate that miR-4293 has oncogenic potential in lung carcinoma.

\section{Knockdown of WFDC21P Attenuates the Oncogenic Role of miR-4293}

The above results show that miR-4293 exert an oncogenic role and increases WFDC21P expression, which indicates that miR-4293 may exert its oncogenic role by promoting WFDC21P expression. To better understand whether the role of miR-4293 can be attenuated by WFDC21P knockdown, we first evaluated cell proliferation co-influenced by miR-4293 and si-WFDC21P. WFDC21P knockdown suppressed cell proliferation and miR-4293 transfection promoted cell proliferation. More importantly, the increase in cell proliferation resulting from miR-4293 transfection was markedly alleviated by si-WFDC21P (Fig. 6A). The apoptosis assay showed that WFDC21P knockdown promoted apoptosis and also significantly abolished the increased cell viability resulting from miR-4293 transfection (Fig. 6B).

We then examined the expression of Bcl2, anit-apoptosis factors, which was enhanced by miR-4293 transfection and suppressed by WFDC21P knockdown compared with scrambled control. Moreover, Bcl2 elevation caused by miR-4293 treatment was attenuated by WFDC21P knockdown. Besides, miR-4293 transfection with WFDC21P knockdown resulted in lower expression of Bcl2 compared with miR-4293 transfection with scrambled control. The expression of Bax was elevated by WFDC21P knockdown but was suppressed by miR-4293 transfection compared with scrambled control. si-WFDC21P alleviated the 
downregulation of Bax expression affected by miR-4293. Accordingly, WFDC21P knockdown induced more caspase-3 cleaved than miR-4293 treatment. miR-4293 transfection attenuated the elevation of cleavage caspase-3 induced by WFDC21P knockdown. More importantly, we found that miR-4293 transfection enhanced STAT3 phosphorylation, which was alleviated by si-WFDC21P (Fig. 6C). RTCA station analysis was performed to continuously observe cells migration, which gradually increased in miR-4293-treated cells but was blocked by si-WFDC21P treatment (Fig. 6D and E).

To further study whether the oncogenic role of miR-4293 can be attenuated by blocking WFDC21P, we investigated whether ASO-4293 inhibition of lung cancer cell proliferation can be restored by WFDC21P overexpression. We found that the proliferative capacity was suppressed by ASO-4293 inhibitor and enhanced by WFDC21P overexpression compared with control. More interestingly, the inhibition of cell proliferation by ASO-4293 was restored to a certain extent after WFDC21P overexpression (Fig. 6F). Compared with control treatment, ASO-4293 transfection increased apoptosis. Even though WFDC21P overexpression could promote cell viability, the percentage of apoptotic cells was still elevated by ASO4293 transfection (Fig. 6G). By examination of apoptosis related factors, we found that the expression of $\mathrm{Bcl} 2$ was decreased in ASO-4293-transfected cells, but increased in WFDC21P overexpression cells. ASO4293 transfection with WFDC21P overexpressing resulted in attenuation of $\mathrm{Bcl} 2$ expression compared with control treatment. In contrast, Bax expression was significantly enhanced by ASO-4293 transfection. WFDC21P overexpression partially attenuated Bax expression induced by ASO-4293. As expected, WFDC21P overexpression decreased the cleavage of caspase-3 compared with scramble control. And attenuation of caspase-3 cleavage caused by WFDC21P overexpression was alleviated by ASO-4293 treatment. We also found that ASO-4293 resulted in less activated STAT3, while WFDC21P overexpression increased STAT3 activation. WFDC21P overexpression also partially increased STAT3 levels induced by ASO-4293 (Fig. 6H). As expected, ASO-4293 treatment could also alleviate migration capacity by WFDC21P overexpression (Fig. 6I-L). Together, these results indicate that oncogenic activity of miR-4293 can be attenuated by blocking WFDC21P expression.

\section{WFDC21P and MiR-4293 Promote Tumorigenesis in vivo.}

Both miR-4293 and WFDC21P have oncogenic activities in lung carcinoma cells. To further investigate the roles of miR-4293 and WFDC21P in vivo, we hypodermically injected A549 cells stably treated with plasmid, or miRNA, or siRNA, or controls into nude mice to produce xenografts. Every 3 days, we measured the volumes of the xenografts. One month after injection, the mice were sacrificed. Stably expression of WFDC21P resulted in enhanced tumor growth both in volumes and weights compared with pcDNA control (Fig. 7A-C). We examined expression of WFDC21P and STAT3 phosphorylation in the xenografts. As expected, WFDC21P overexpression in injected xenografts resulted in elevation of WFDC21P and STAT3 phosphorylation (Figure S 4A and B).

We then evaluated the effects of miR-4293 or WFDC21P knockdown on xenograft growth. miR-4293 treatment enhanced growth in both volumes and weights of xenografts compared with scrambled 
control. In contrast, WFDC21P knockdown resulted in xenografts growth that was significantly lower than that produced by miR-4293 overexpression and also than scrambled control (Fig. 7D-F). Compared with scrambled control, miR-4293 treatment resulted in elevation of miR-4293, WFDC21P and STAT3 phosphorylation in xenografts. Conversely, these measures were attenuated by WFDC21P knockdown (Figure S4C-E).

To better understand the cooperative action of miR-4293 and WFDC21P in tumor growth, we established xenografts with A549 cells co-treated with miR-4293 and scrambled control, miR-4293 and ASO-4293, as well as miR-4293 and siRNA-WFDC21P. As expected, xenografts with miR-4293 and scrambled cotreatment had the highest growth rate of these three groups. Interestingly, ASO-4293 and si-WFDC21P could abolish the tumor promoting effect of miR-4293 almost equally (Fig. 7G-I). Moreover, ASO-4293 attenuated the decrease in WFDC21P or STAT3 levels in xenografts by miR-4293 and si-WFDC21P (Figure S4F-H). Similarly, tumor promotion resulting from WFDC21P stable overexpression was also alleviated by ASO-4293 treatment (Fig. 7J-L). ASO-4293 treatment could also alleviate elevation of WFDC21P and STAT3 phosphorylation in xenografts resulting from WFDC21P overexpression in injected cells (Figure S4I and J). The above results support the ability of miR-4293 and WFDC21P to promote tumor growth in vivo.

\section{Discussion}

IncRNAs are involved in cell proliferation, metastasis and apoptosis, and can also play oncogenic or tumor suppressive roles. Here, we show that WFDC21P is significantly elevated in NSCLC tissues and promotes tumor cell proliferation and metastasis but suppresses apoptosis. DCP2 can directly bind to WFDC21P and promote its downregulation. More interestingly, DCP2 is found to be a target of miR-4293 and down-regulated by miR-4293. It has been reported STAT3 is a target gene of WFDC21P.[23] Importantly, our results confirm that miR-4293 enhances the expression of WFDC21P and promotes STAT3 phosphorylation by a novel mechanism in directly regulating DCP2. In addition, WFDC21P elevates miR-4293 expression by promoting STAT3 activation. As a result of elevated STAT3 activity, apoptosis is suppressed and proliferation is enhanced through Bcl2 elevation, Bax2 attenuation and reduced caspase cleavage (Fig. 8). Together, these findings provide evidence that miR-4293 plays an oncogenic role in NSCLC by suppressing DCP2-mediated WFDC21P degradation.

Accumulated studies have indicated that the prominent class of IncRNA share a common function in negatively impacting on miRNA-mediated gene regulation.[36, 37] Interestingly, we found that, in contrast to ceRNA, not only WFDC21P could be up-regulated by miR-4293, but miR-4293 expression could be enhanced by WFDC21P through positive feedback. Both WFDC21P and miR-4293 play oncogenic roles in NSCLC. To better understand the mechanism, a key step is to determine miR-4293 targets and we revealed DCP2 to be such a target. As a critical component of the decapping complex, DCP2 is responsible for RNA degradation.[38, 39] By decapping a subset of mRNAs, DCP2 plays an important role in cancer pathogenesis by affecting processes such as cell migration and apoptosis. [40-44] More interestingly, DCP2 is regulated at the post-transcriptional level rather than being transcriptionally 
silenced, and miRNAs are an effective way to down-regulate DCP2. DCP2 mRNA has a fairly long 3'-UTR of $7.2 \mathrm{~kb}$ that may contain many potential miRNA binding sites. miR-141-3p/200a-3p may be involved in repression of DCP2 expression during renal development; therefore, miRNAs can not only directly suppress the expression of target genes by binding the 3'-UTR but can also regulate gene expression by involving DCP2 activity. As a novel finding, we observed that miR-4293 can bind to DCP2 transcripts and down-regulate their expression. Several studies indicate that DCP2 appears to target distinct transcripts and the reported targets are almost all mRNAs. $[40,45,46]$ However, we observed that DCP2 can bind to WFDC21P, a long non-coding RNA, to participate in cancer pathogenesis. We further clarified that WFDC21P degradation played important roles in the tumorigenesis of lung carcinoma, and showed that DCP2 directly regulated by miR-4293, down-regulated WFDC21P expression. The downregulation of IncRNAs mediated by DCP2 may become a novel underlying mechanism of IncRNAs in different kinds of carcinoma. However, DCP2 is not the only one component of the decapping enzyme [47], and the specific underlying mechanism for DCP2 promoting WFDC21P degradation needs further study.

Although we did not confirm a direct interaction between WFDC21P and miR-4293, WFDC21P expression can still be elevated by miR-4293. More importantly, miR-4293 upregulated WFDC21P in both NSCLC cells and xenografts. In addition, the expression of miR-4293 was positively correlated with WFDC21P levels in lung carcinoma. Hence, the elevation of miR-4293 in NSCLC tissues might account for the increase in WFDC21P levels. Recent studies reveal that IncRNAs, as competing endogenous RNA, can bind to related miRNAs to further suppress the function of the miRNAs.[14, 48] However, we found WFDC21P could elevate miR-4293 levels via a positive feedback loop. Furthermore, the oncogenic role of miR-4293 was blocked by suppressing WFDC21P. miRNAs have the potential to target hundreds of mRNAs because of imperfect complementarity when binding. Different targets result in different roles of miRNAs.[49-51] Although WFDC21P did not directly interact with miR-4293, we found that WFDC21P may play a major role in the function of miR-4293, and WFDC21P can be considered as a downstream regulator of the miR4293 signaling pathway. In the present study, we observed that WFDC21P knockdown almost completely abolished the effects of miR-4293 on proliferation, apoptosis and metastasis. WFDC21P overexpression could only restore the effects of ASO-4293 suppression to a certain extent, possibly because of DCP2 activation. Transfection of ASO-4293 almost abolished suppression of DCP2 by miR-4293, and DCP2 activation may be greater with ASO-4293 treatment. Even though WFDC21P was overexpressed, its degradation was upregulated by activated DCP2.

STAT3 plays a critical role in many pathophysiological processes, including cancer cell proliferation, antiapoptosis and metastasis.[52, 53] We found that STAT3 expression was not only elevated in lung carcinoma, but also positively correlated with the expression of both WFDC21P and miR-4293. In addition to enhancing STAT3 activation, WFDC21P possibly participates in the regulation of STAT3 signaling.[54, 55] As a regulator of WFDC21P, miR-4293 could further elevate STAT3 phosphorylation. Moreover, by regulating STAT3 activation, WFDC21P could elevate the expression of miR-4293. Hence, STAT3 may play a critical role in the proto-oncogenic capacity of WFDC21P and miR-4293. STAT3 deactivation may suppress the function of WFDC21P and miR-4293. 


\section{Conclusions}

Many IncRNAs, as competing endogenous factors, have been reported to participate into cancer pathogenesis by suppressing the function of miRNAs. Differently, this study demonstrates a novel interaction pattern between IncRNA and miRNA, and we found that miR-4293 up-regulates the expression of WFDC21P through its degradation by directly mediating DCP2, which plays important roles in the tumorigenesis of lung carcinoma. Moreover, WFDC21P interference almost completely abolished the effects of miR-4293 on the proliferation of lung carcinoma cells, which indicates that WFDC21P knockdown might be an effective strategy for lung cancer therapy. The present study provided a valuable theoretical basis for the discovery of lung carcinoma therapeutic targets and diagnosis markers based on WFDC21P and miR-4293.

\section{Abbreviations}

IncRNA WFDC21P: WFDC21P; DCP2: decapping enzyme 2; NSCLC: non-small cell lung cancer; RIP: RNA immunoprecipitation; ASO: antisense oligonucleotides; UTR: untranslated region;

\section{Declarations}

\section{Ethics approval and consent to participate}

All animal experiments were approved by the Committee on the Ethics of Animal Experiments of Binzhou Medical University and performed in accordance with the National Institutes of Health guide for the care and use of Laboratory animals. The experiments were performed in accordance with the relevant guidelines of the Code of Ethics of the World Medical Association for experiments involving humans and the Medical Ethics Committee of Binzhou Medical University. Patients provided written informed consent and the study procedures were fully explained before study inclusion. The authors confirmed that we have obtained written consent from the patients to publish this manuscript.

\section{Consent for publication}

The authors confirmed that we have obtained written consent from the patients to publish this manuscript.

\section{Availability of data and materials}

All data generated during this study are included either in article or in the additional files.

\section{Declaration of Interests}

The authors declare that they have no conflicts of interest concerning this article.

\section{Funding}


The present study was supported by the National Natural Science Foundation of China (No.81772281, 31371321, 81702296), the Shandong Science and Technology Committee (No. ZR2019MH022, 2018GSF118056, 2017GSF18124), the Yantai Science and Technology Committee (2018XSCC051)区the Education Department of Shandong Province (2019KJK014), and the Shandong Province Taishan Scholar Project (no. ts201712067).

\section{Authors' Contributions}

Conception and design: SYX

Acquisition of data (provided animals, acquired and managed patients, provided facilities, etc.): QZ, YFY, QL, YJL, PYW, NX

Analysis and interpretation of data (e.g., statistical analysis, biostatistics, computational analysis): QZ, PYW, SYX

Writing, review, and/or revision of the manuscript: SYX, YFY, QZ, CZ, PYW

Administrative, technical, or material support (i.e., reporting or organizing data, constructing databases): QZ, YFY, QL, YJL, PYW, LP, JXH, RRW, GBS, NX, YRL, BCT, FJ, SX

Study supervision: SYX, PYW

\section{Acknowledgements}

We thank Jeremy Allen, PhD, from Liwen Bianji, Edanz Group China (www.liwenbianji.cn/ac), for editing the English text of a draft of this manuscript.

\section{References}

1. Herbst RS, Heymach JV, Lippman SM: Lung cancer. N Engl J Med 2008, 359:1367-1380.

2. Swanton C, Govindan R: Clinical Implications of Genomic Discoveries in Lung Cancer. N Engl J Med 2016, 374:1864-1873.

3. Serghiou S, Kyriakopoulou A, loannidis JP: Long noncoding RNAs as novel predictors of survival in human cancer: a systematic review and meta-analysis. Mol Cancer 2016, 15:50.

4. Du L, Schageman JJ, Irnov, Girard L, Hammond SM, Minna JD, Gazdar AF, Pertsemlidis A: MicroRNA expression distinguishes SCLC from NSCLC lung tumor cells and suggests a possible pathological relationship between SCLCs and NSCLCs. J Exp Clin Cancer Res 2010, 29:75.

5. Sarfi M, Abbastabar M, Khalili E: Long noncoding RNAs biomarker-based cancer assessment. J Cell Physiol 2019, 234:16971-16986.

6. Zhang J, Lin Z, Gao Y, Yao T: Downregulation of long noncoding RNA MEG3 is associated with poor prognosis and promoter hypermethylation in cervical cancer. J Exp Clin Cancer Res 2017, 36:5. 
7. Long X, Song K, Hu H, Tian Q, Wang W, Dong Q, Yin X, Di W: Long non-coding RNA GAS5 inhibits DDP-resistance and tumor progression of epithelial ovarian cancer via GAS5-E2F4-PARP1-MAPK axis. J Exp Clin Cancer Res 2019, 38:345.

8. Zhang S, Zheng F, Zhang L, Huang Z, Huang X, Pan Z, Chen S, Xu C, Jiang Y, Gu S, et al: LncRNA HOTAIR-mediated MTHFR methylation inhibits 5-fluorouracil sensitivity in esophageal cancer cells. $J$ Exp Clin Cancer Res 2020, 39:131.

9. Lin N, Yao Z, Xu M, Chen J, Lu Y, Yuan L, Zhou S, Zou X, Xu R: Long noncoding RNA MALAT1 potentiates growth and inhibits senescence by antagonizing ABI3BP in gallbladder cancer cells. $J$ Exp Clin Cancer Res 2019, 38:244.

10. Yao RW, Wang Y, Chen LL: Cellular functions of long noncoding RNAs. Nat Cell Biol 2019, 21:542551.

11. Salmena L, Poliseno L, Tay Y, Kats L, Pandolfi PP: A ceRNA hypothesis: the Rosetta Stone of a hidden RNA language? Cell 2011, 146:353-358.

12. Bartonicek N, Maag JL, Dinger ME: Long noncoding RNAs in cancer: mechanisms of action and technological advancements. Mol Cancer 2016, 15:43.

13. Tang Y, Cheung BB, Atmadibrata B, Marshall GM, Dinger ME, Liu PY, Liu T: The regulatory role of long noncoding RNAs in cancer. Cancer Lett 2017, 391:12-19.

14. Braconi C, Kogure T, Valeri N, Huang N, Nuovo G, Costinean S, Negrini M, Miotto E, Croce CM, Patel T: microRNA-29 can regulate expression of the long non-coding RNA gene MEG3 in hepatocellular cancer. Oncogene 2011, 30:4750-4756.

15. Jonas S, Izaurralde E: Towards a molecular understanding of microRNA-mediated gene silencing. Nat Rev Genet 2015, 16:421-433.

16. Brase JC, Wuttig D, Kuner R, Sultmann H: Serum microRNAs as non-invasive biomarkers for cancer. Mol Cancer 2010, 9:306.

17. Wu H, Li L, Chen KM, Homolka D, Gos P, Fleury-Olela F, McCarthy AA, Pillai RS: Decapping Enzyme NUDT12 Partners with BLMH for Cytoplasmic Surveillance of NAD-Capped RNAs. Cell Rep 2019, 29:4422-4434 e4413.

18. Wang $Y$, Wang $L$, Chen $C$, Chu $X$ : New insights into the regulatory role of microRNA in tumor angiogenesis and clinical implications. Mol Cancer 2018, 17:22.

19. Scholzova E, Malik R, Sevcik J, Kleibl Z: RNA regulation and cancer development. Cancer Lett 2007, 246:12-23.

20. Geisler S, Lojek L, Khalil AM, Baker KE, Coller J: Decapping of long noncoding RNAs regulates inducible genes. Mol Cell 2012, 45:279-291.

21. Ma Q, Wang Q, Zhong D: [Advances of human epididymis protein 4 in lung cancer]. Zhongguo Fei Ai Za Zhi 2015, 18:184-186.

22. Speeckaert MM, Speeckaert R, Delanghe JR: Human epididymis protein 4 in cancer diagnostics: a promising and reliable tumor marker. Adv Clin Chem 2013, 59:1-21. 
23. Wang P, Xue Y, Han Y, Lin L, Wu C, Xu S, Jiang Z, Xu J, Liu Q, Cao X: The STAT3-binding long noncoding RNA Inc-DC controls human dendritic cell differentiation. Science 2014, 344:310-313.

24. Li XX, Liu YM, Li YJ, Xie N, Yan YF, Chi YL, Zhou L, Xie SY, Wang PY: High glucose concentration induces endothelial cell proliferation by regulating cyclin-D2-related miR-98. J Cell Mol Med 2016, 20:1159-1169.

25. Zhang YX, Yan YF, Liu YM, Li YJ, Zhang HH, Pang M, Hu JX, Zhao W, Xie N, Zhou L, et al: Smad3related miRNAs regulated oncogenic TRIB2 promoter activity to effectively suppress lung adenocarcinoma growth. Cell Death Dis 2016, 7:e2528.

26. Lv Q, Hu JX, Li YJ, Xie N, Song DD, Zhao W, Yan YF, Li BS, Wang PY, Xie SY: MiR-320a effectively suppresses lung adenocarcinoma cell proliferation and metastasis by regulating STAT3 signals. Cancer Biol Ther 2017, 18:142-151.

27. Wang PY, Sun YX, Zhang S, Pang M, Zhang HH, Gao SY, Zhang C, Lv CJ, Xie SY: Let-7c inhibits A549 cell proliferation through oncogenic TRIB2 related factors. FEBS Lett 2013, 587:2675-2681.

28. Bingle L, Cross SS, High AS, Wallace WA, RassI D, Yuan G, Hellstrom I, Campos MA, Bingle CD: WFDC2 (HE4): a potential role in the innate immunity of the oral cavity and respiratory tract and the development of adenocarcinomas of the lung. Respir Res 2006, 7:61.

29. McAlhany SJ, Ressler SJ, Larsen M, Tuxhorn JA, Yang F, Dang TD, Rowley DR: Promotion of angiogenesis by ps20 in the differential reactive stroma prostate cancer xenograft model. Cancer Res 2003, 63:5859-5865.

30. Wu WF, Maneix L, Insunza J, Nalvarte I, Antonson P, Kere J, Yu NY, Tohonen V, Katayama S, Einarsdottir $\mathrm{E}$, et al: Estrogen receptor beta, a regulator of androgen receptor signaling in the mouse ventral prostate. Proc Natl Acad Sci U S A 2017, 114:E3816-E3822.

31. Smebye ML, Agostini A, Johannessen B, Thorsen J, Davidson B, Trope CG, Heim S, Skotheim RI, Micci F: Involvement of DPP9 in gene fusions in serous ovarian carcinoma. BMC Cancer 2017, 17:642.

32. He YP, Li LX, Tang JX, Yi L, Zhao Y, Zhang HW, Wu ZJ, Lei HK, Yu HQ, Nian WQ, Gan L: HE4 as a biomarker for diagnosis of lung cancer: A meta-analysis. Medicine (Baltimore) 2019, 98:e17198.

33. Madar S, Brosh R, Buganim Y, Ezra O, Goldstein I, Solomon H, Kogan I, Goldfinger N, Klocker H, Rotter V: Modulated expression of WFDC1 during carcinogenesis and cellular senescence. Carcinogenesis 2009, 30:20-27.

34. Bouchard D, Morisset D, Bourbonnais Y, Tremblay GM: Proteins with whey-acidic-protein motifs and cancer. Lancet Oncol 2006, 7:167-174.

35. Ozgur S, Chekulaeva M, Stoecklin G: Human Pat1b connects deadenylation with mRNA decapping and controls the assembly of processing bodies. Mol Cell Biol 2010, 30:4308-4323.

36. Chan JJ, Tay Y: Noncoding RNA:RNA Regulatory Networks in Cancer. Int J Mol Sci 2018, 19.

37. Jiang R, Hu C, Li Q, Cheng Z, Gu L, Li H, Guo Y, Li Q, Lu Y, Li K, et al: Sodium new houttuyfonate suppresses metastasis in NSCLC cells through the Linc00668/miR-147a/slug axis. J Exp Clin Cancer Res 2019, 38:155. 
38. Dunckley T, Parker R: The DCP2 protein is required for mRNA decapping in Saccharomyces cerevisiae and contains a functional MutT motif. EMBO J 1999, 18:5411-5422.

39. Mugridge JS, Ziemniak M, Jemielity J, Gross JD: Structural basis of mRNA-cap recognition by Dcp1Dcp2. Nat Struct Mol Biol 2016, 23:987-994.

40. Zeidan Q, He F, Zhang F, Zhang H, Jacobson A, Hinnebusch AG: Conserved mRNA-granule component Scd6 targets Dhh1 to repress translation initiation and activates Dcp2-mediated mRNA decay in vivo. PLoS Genet 2018, 14:e1007806.

41. Mencia N, Selga E, Noe V, Ciudad CJ: Underexpression of miR-224 in methotrexate resistant human colon cancer cells. Biochem Pharmacol 2011, 82:1572-1582.

42. Gaviraghi M, Vivori C, Pareja Sanchez Y, Invernizzi F, Cattaneo A, Santoliquido BM, Frenquelli M, Segalla S, Bachi A, Doglioni C, et al: Tumor suppressor PNRC1 blocks rRNA maturation by recruiting the decapping complex to the nucleolus. EMBO J 2018, 37.

43. Mugridge JS, Gross JD: Decapping enzymes STOP "cancer" ribosomes in their tracks. EMBO J 2018 , 37.

44. Wojtczak BA, Sikorski PJ, Fac-Dabrowska K, Nowicka A, Warminski M, Kubacka D, Nowak E, Nowotny M, Kowalska J, Jemielity J: 5'-Phosphorothiolate Dinucleotide Cap Analogues: Reagents for Messenger RNA Modification and Potent Small-Molecular Inhibitors of Decapping Enzymes. J Am Chem Soc 2018, 140:5987-5999.

45. Li Y, Song M, Kiledjian M: Differential utilization of decapping enzymes in mammalian mRNA decay pathways. RNA 2011, 17:419-428.

46. Kramer S, McLennan AG: The complex enzymology of mRNA decapping: Enzymes of four classes cleave pyrophosphate bonds. Wiley Interdiscip Rev RNA 2019, 10:e1511.

47. Kakumani PK, Harvey LM, Houle F, Guitart T, Gebauer F, Simard MJ: CSDE1 controls gene expression through the miRNA-mediated decay machinery. Life Sci Alliance 2020, 3.

48. Guttman M, Rinn JL: Modular regulatory principles of large non-coding RNAs. Nature 2012, 482:339346.

49. Mohr AM, Mott JL: Overview of microRNA biology. Semin Liver Dis 2015, 35:3-11.

50. Saliminejad K, Khorram Khorshid HR, Soleymani Fard S, Ghaffari SH: An overview of microRNAs: Biology, functions, therapeutics, and analysis methods. J Cell Physiol 2019, 234:5451-5465.

51. Shah MY, Ferrajoli A, Sood AK, Lopez-Berestein G, Calin GA: microRNA Therapeutics in Cancer - An Emerging Concept. EBioMedicine 2016, 12:34-42.

52. Lee JJ, Kim HJ, Yang CS, Kyeong HH, Choi JM, Hwang DE, Yuk JM, Park K, Kim YJ, Lee SG, et al: A high-affinity protein binder that blocks the IL-6/STAT3 signaling pathway effectively suppresses nonsmall cell lung cancer. Mol Ther 2014, 22:1254-1265.

53. Yeung YT, Yin S, Lu B, Fan S, Yang R, Bai R, Zhang C, Bode AM, Liu K, Dong Z: Losmapimod Overcomes Gefitinib Resistance in Non-small Cell Lung Cancer by Preventing Tetraploidization. EBioMedicine 2018, 28:51-61. 
54. Alikhah A, Pahlevan Kakhki M, Ahmadi A, Dehghanzad R, Boroumand MA, Behmanesh M: The role of Inc-DC long non-coding RNA and SOCS1 in the regulation of STAT3 in coronary artery disease and type 2 diabetes mellitus. J Diabetes Complications 2018, 32:258-265.

55. Zhuang L, Tian J, Zhang X, Wang H, Huang C: Lnc-DC regulates cellular turnover and the HBVinduced immune response by TLR9/STAT3 signaling in dendritic cells. Cell Mol Biol Lett 2018, 23:43.

\section{Figures}
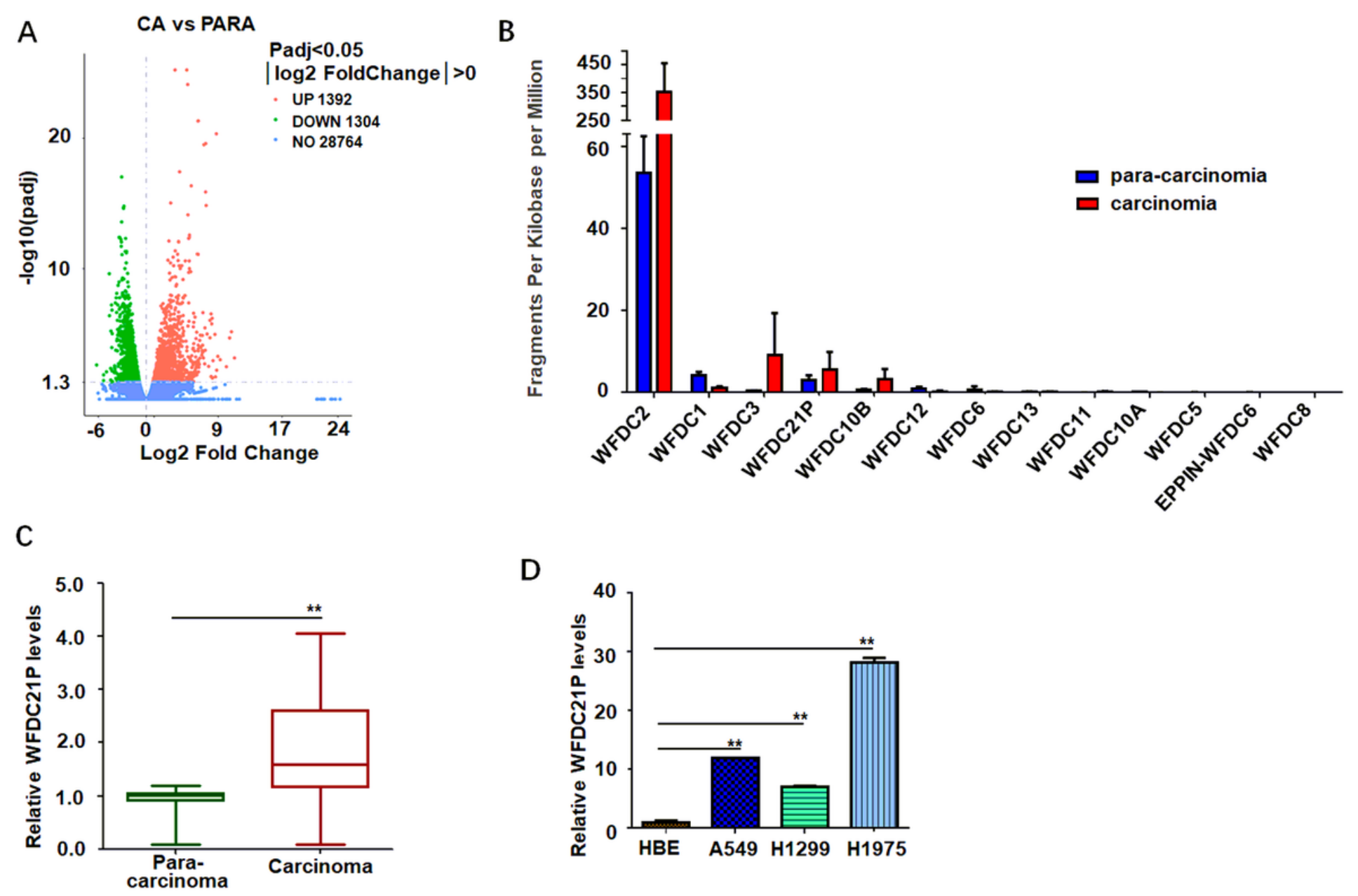

Figure 1

WFDC21P Increases in Lung Carcinoma Cells and Tissues. (A) Vocalno Plot indicated up and down regulated mRNAs in lung carcinoma tissues compared with para-carcinoma tissue. (B) FPKM analysis from RNA-seq dataset showed the expression of WFDC family members in carcinoma tissues compared with para-carcinoma tissue. (C) qRT-PCR analysis showed WFDC21P expression in NSCLC tissues and corresponding para-carcinoma tissues $(n=16)$. Data are expressed as median (interquartile range). **P<0.01; Mann-Whitney U test. (D) qRT-PCR analysis showed WFDC21P expression in A549, H1299, $\mathrm{H} 1975$ and HBE cells. Data are expressed as mean \pm SD for triplicate experiments. ${ }^{* *} p<0.01$; ANOVA test. 
A

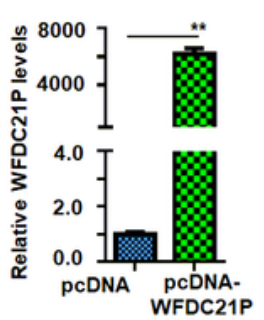

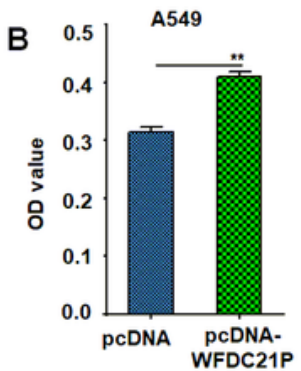

C

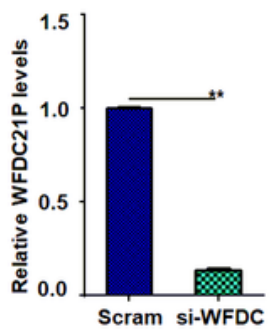

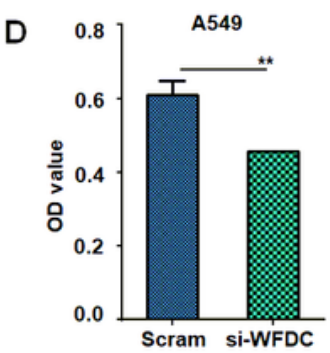

E
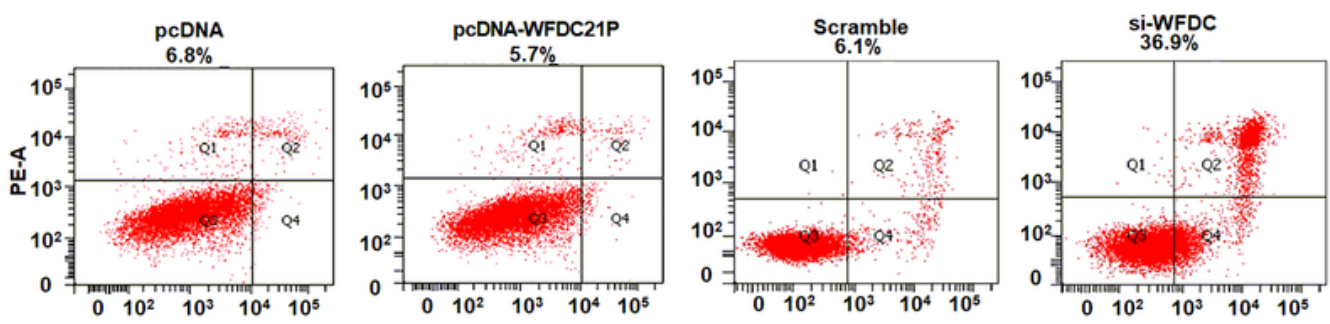

FITC

F
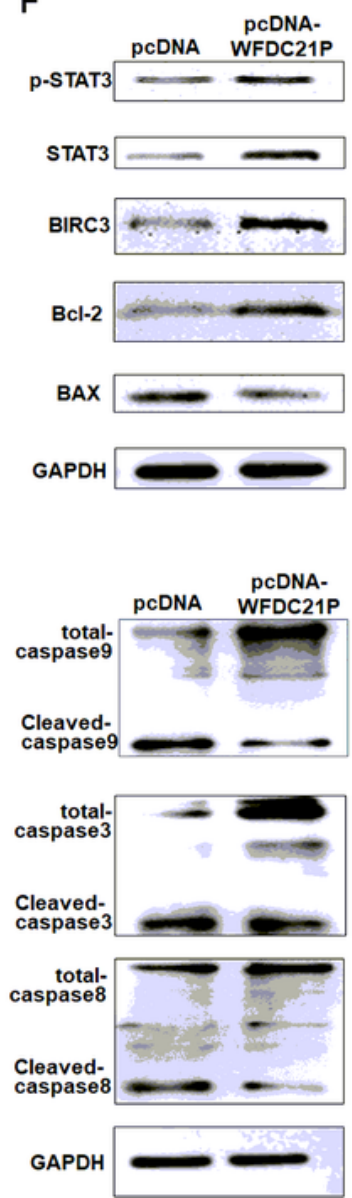
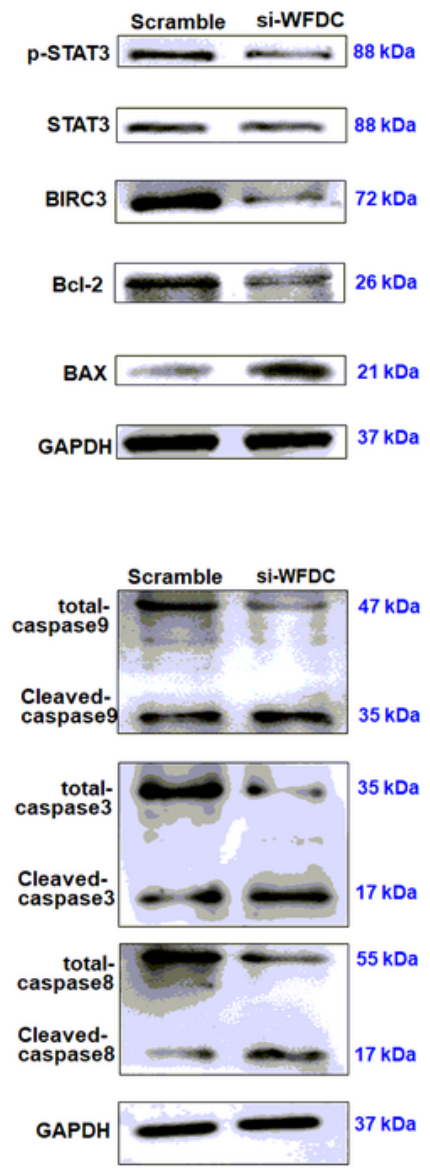

G
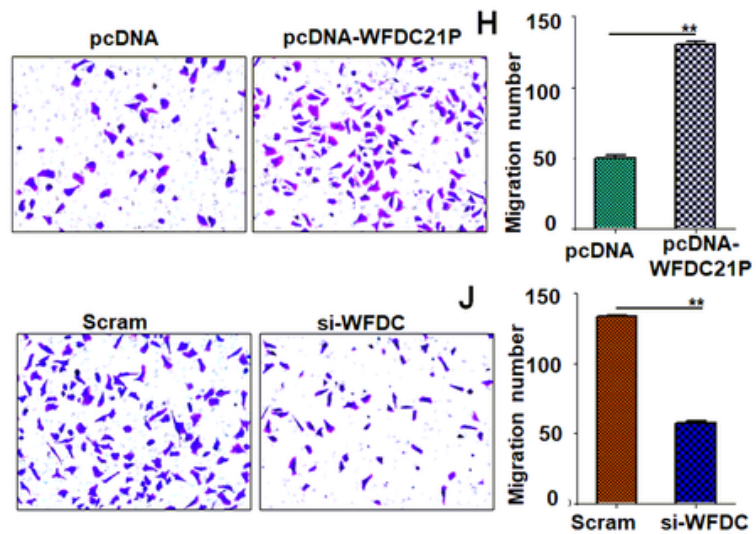

$\mathrm{K}$
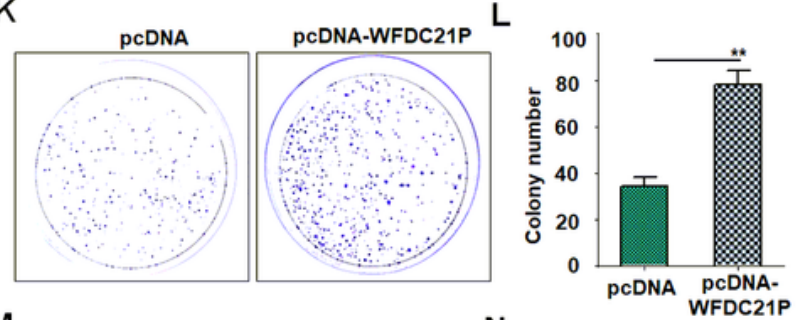

M

si-WFDC

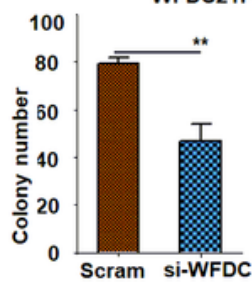

Figure 2

WFDC21P Enhances Cell Proliferation and Migration. (A) qRT-PCR analysis showed WFDC21P expression in pcDNA-WFDC21P- and pcDNA-treated cells. Data are expressed as mean \pm SD for triplicate experiments. ${ }^{* *} p<0.01$; Student's $t$ test. (B) MTT assays showed proliferation of A549 at $24 \mathrm{~h}$ posttransfection of pcDNA-WFDC21P and pc-DNA. Data are expressed as mean \pm SD for triplicate experiments. ${ }^{*} \mathrm{p}<0.01$; Student's $t$ test. (C) qRT-PCR analysis showed WFDC21P expression in si- 
WFDC21P- and scram-treated cells. Data are expressed as mean \pm SD for triplicate experiments. ** $p<0.01$; Student's t test. (D) MTT assays showed proliferation of A549 at $24 \mathrm{~h}$ post-transfection of siWFDC21P and scramble. Data are expressed as mean \pm SD for triplicate experiments. ** $p<0.01$; Student's t test. (E) FACS analysis showed $A 549$ cell apoptosis at $24 \mathrm{~h}$ post-transfection of pc-DNA, pcDNA-WFDC21P, si-WFDC21P and scrambled control. (F) Western blot showed the expression of STAT3 and apoptosis-related factors (Bcl-2, Bax, BIRC3, and Caspase 3,8,9) in A549 cells. pcDNA vs pcDNAWFDC21P; scramble vs si-WFDC. (G-J) Transwell assays showed A549 migration. (G) and (H) pcDNA vs pcDNA-WFDC21P; $(\mathrm{I})$ and $(\mathrm{J})$ scramble vs si-WFDC. Data are expressed as mean \pm SD for triplicate experiments. ${ }^{* *} p<0.01$; Student's $t$ test. (K-N) Colony formation assays of A549. (K) and (L) pcDNA vs pcDNA-WFDC21P; $(M)$ and $(N)$ scramble vs si-WFDC. Data are expressed as mean \pm SD for triplicate experiments. ${ }^{*} \mathrm{p}<0.01$; Student's t test.

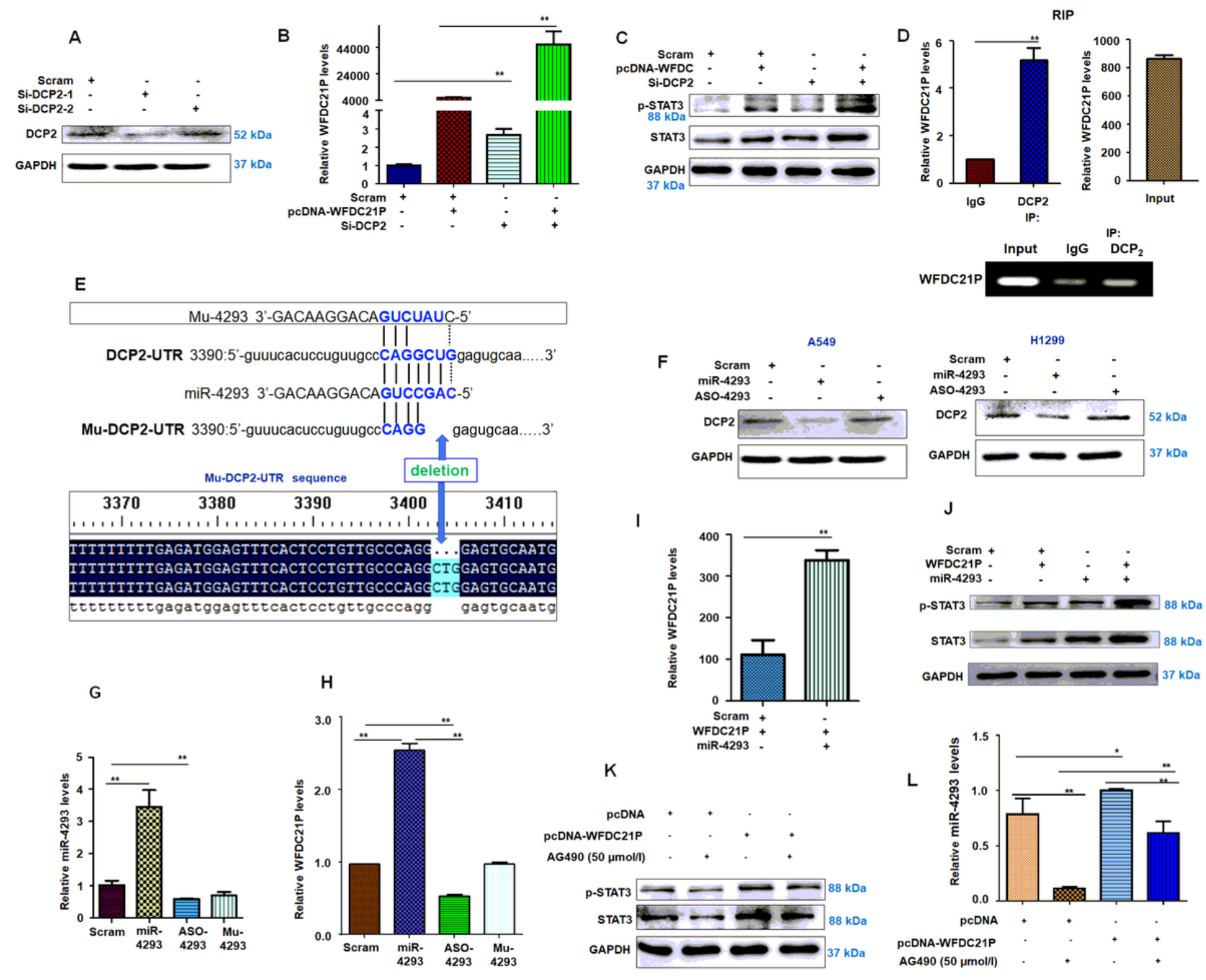

Figure 3 
WFDC21P Interacts with, and is regulated by DCP2 which is a target of miR-4293. (A) Western blot showed DCP2 expression in siRNA-DCP2-1-, siRNA-DCP2-2- or scramble-treated A549 cells. (B) qRT-PCR analysis showed WFDC21P expression in pcDNA-WFDC21P+scramble-, si-DCP2-, si-DCP2+pcDNAWFDC21P- or scramble-treated A549 cells. Data are expressed as mean \pm SD for triplicate experiments. ** $\mathrm{p}<0.01$; ANOVA test. (C) Western blot showed STAT3 phosphorylation in pcDNA-WFDC21P+scramble-, siDCP2-, si-DCP2+pcDNA-WFDC21P- or scramble-treated A549 cells. (D) RIP assay suggested the interaction between WFDC21P and DCP2. Upper panel, qRT-PCR analysis of WFDC21P pull-down by IgG or DCP2; lower panel, electrophoresis for WFDC21P pull-down by IgG or DCP2 after PCR amplification. Data are expressed as mean \pm SD for triplicate experiments. ${ }^{* \star} p<0.01$; Student's $t$ test. (E) TargetScan analysis predicted that the DCP2 mRNA-3'-UTR is targeted by miR-4293. We synthesized a mutant miR4293 (Mu-4293) and cloned DCP2 mRNA-3'-UTR and mu-DCP2-UTR sequences. (F) Western blot showed the expression of DCP2 in miR-4293-, ASO-4293- or scramble-treated A549 cells (left) and H1299 cells (right). (G) qRT-PCR analysis showed miR-4293 levels in miR-4293-, ASO-4293, Mu-4293 or scrambletreated A549 cells. Data are expressed as mean \pm SD for triplicate experiments. ${ }^{* \star} p<0.01$; ANOVA test. (H) qRT-PCR analysis showed WFDC21P expression in miR-4293-, ASO-4293, Mu-4293 or scrambletreated A549 cells. Data are expressed as mean \pm SD for triplicate experiments. ${ }^{*} p<0.01$; ANOVA test. (I) qRT-PCR analysis showed WFDC21P expression in miR-4293+pcDNA-WFDC21P- or scramble+ pcDNAWFDC21P-treated A549 cells. Data are expressed as mean \pm SD for triplicate experiments. ${ }^{*} p<0.01$; Student's $t$ test. (J) Western blot showed STAT3 phosphorylation in pcDNA-WFDC21P+scramble-, miR4293-, miR-4293+pcDNA-WFDC21P- or scramble-treated A549 cells. (K) Western blot showed STAT3 phosphorylation in AG490+ pcDNA-, pcDNA-WFDC21P-, AG490+ pcDNA-WFDC21P- or pcDNA treated A549 cells. (L) qRT-PCR analysis showed miR-4293 expression in AG490+ pcDNA-, pcDNA-WFDC21P-, AG490 + pcDNA-WFDC21P- or pcDNA treated A549 cells. Data are expressed as mean \pm SD for triplicate experiments. ${ }^{* *} \mathrm{p}<0.01,{ }^{*} \mathrm{P}<0.05$; ANOVA test.
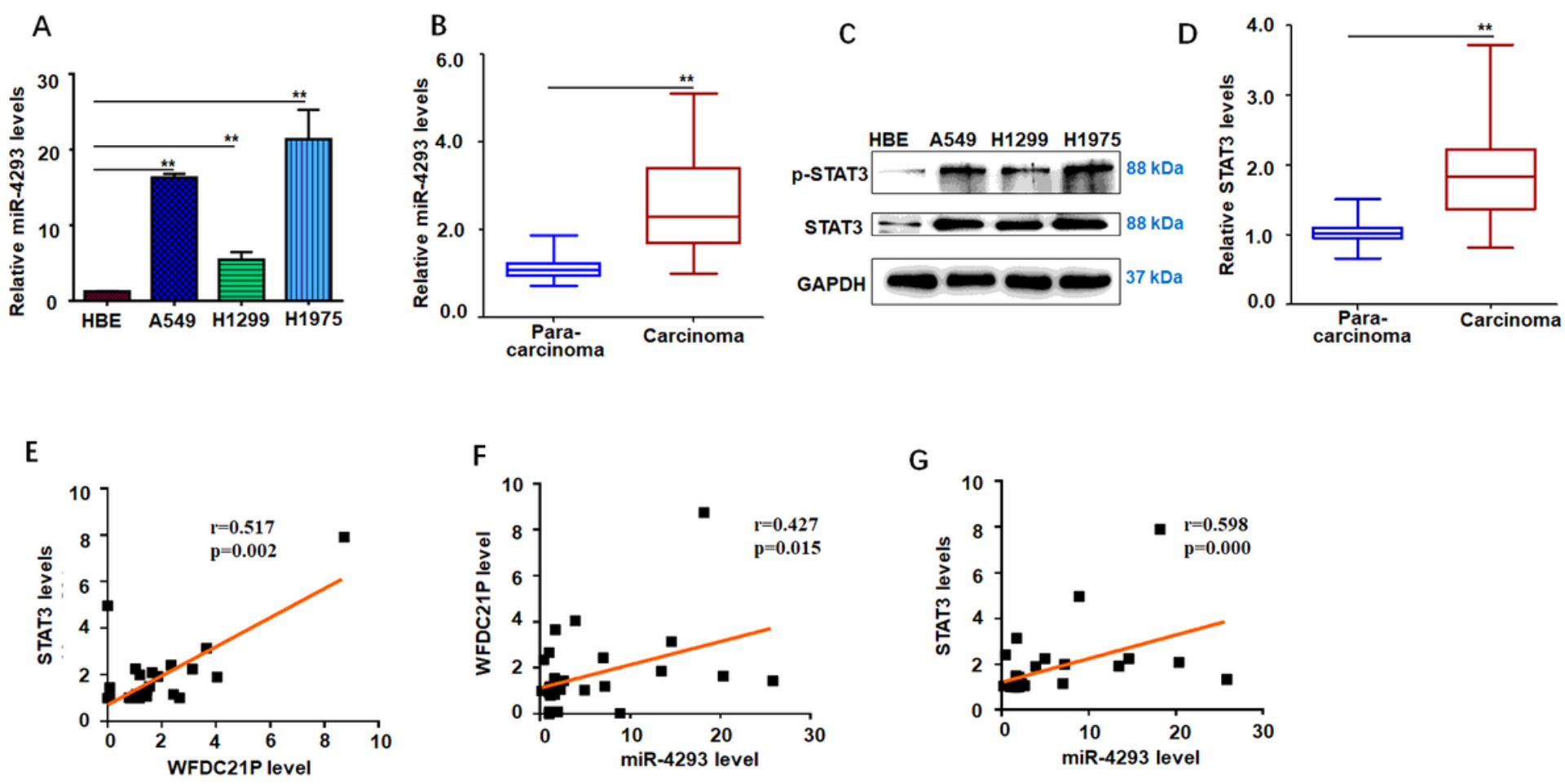


\section{Figure 4}

MiR-4293 and STAT3 Levels also Increased in NSCLC Cells and Tissues. (A) qRT-PCR analysis showed miR-4293 expression in A549, H1299, H1975 and HBE cells. Data are expressed as mean \pm SD for triplicate experiments. ** $p<0.01$; ANOVA test. (B) qRT-PCR analysis showed miR-4293 expression in NSCLC tissues and corresponding para-carcinoma tissues $(n=16)$. Data are expressed as median (interquartile range). ${ }^{*} \mathrm{P}<0.01$; Mann-Whitney U test. (C) Western blot showed phosphorylation of STAT3 in A549, H1299, H1975 and HBE cells. (D) qRT-PCR analysis showed STAT3 expression in NSCLC tissues and corresponding para-carcinoma tissues $(n=16)$. Data are expressed as median (interquartile range). **P<0.01; Mann-Whitney U test. (E-G) Pearson's correlation analysis of miR-4293 expression and WFDC21P expression ( $n=16, * * P=0.015)(E)$, miR-4293 expression and STAT3 expression $(n=16$, $\star * P<0.01)(F)$, WFDC21P expression and STAT3 expression $(n=16, * * P<0.01)(G)$.
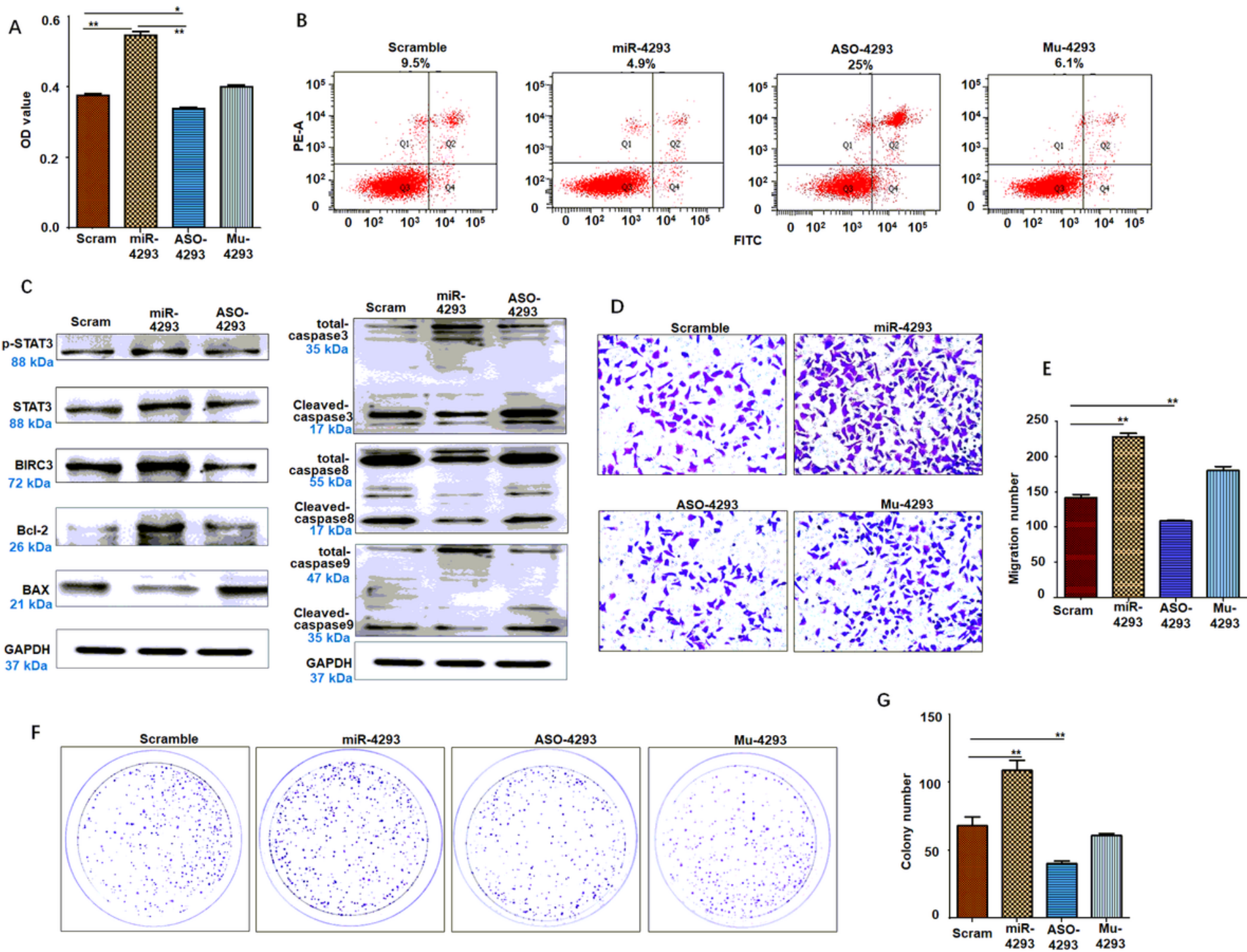

\section{Figure 5}

MiR-4293 Promotes Cell Proliferation and Migration (A) MTT assay showed proliferation of A549 at $24 \mathrm{~h}$ post-transfection of miR-4293, ASO-4293, mu-4293 or scrambled. Data are expressed as mean \pm SD for 
triplicate experiments. ** $p<0.01, * p<0.05$; ANOVA test. (B) FACS analysis showed A549 cell apoptosis at $24 \mathrm{~h}$ post-transfection of miR-4293, ASO-4293, mu-4293 or scrambled. (C) Western blot showed expression of STAT3 and apoptosis-related factors (Bcl-2, Bax, BIRC3, and Caspase 3,8,9) in miR-4293-, ASO-4293-, or scramble-treated A549 cells. (D-E) Transwell migration assay of A549 at $24 \mathrm{~h}$ posttransfection of miR-4293, ASO-4293, mu-4293 or scrambled. Data are expressed as mean \pm SD for triplicate experiments. ${ }^{*} \mathrm{p}<0.01$; ANOVA test. (F-G) Colony formation assays of A549 at $24 \mathrm{~h}$ posttransfection of miR-4293, ASO-4293, mu-4293 or scrambled. Data are expressed as mean \pm SD for triplicate experiments. ${ }^{*} \mathrm{p}<0.01$; ANOVA test. 


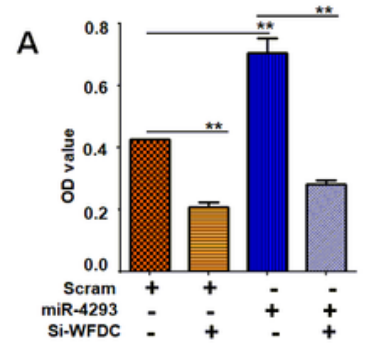

D
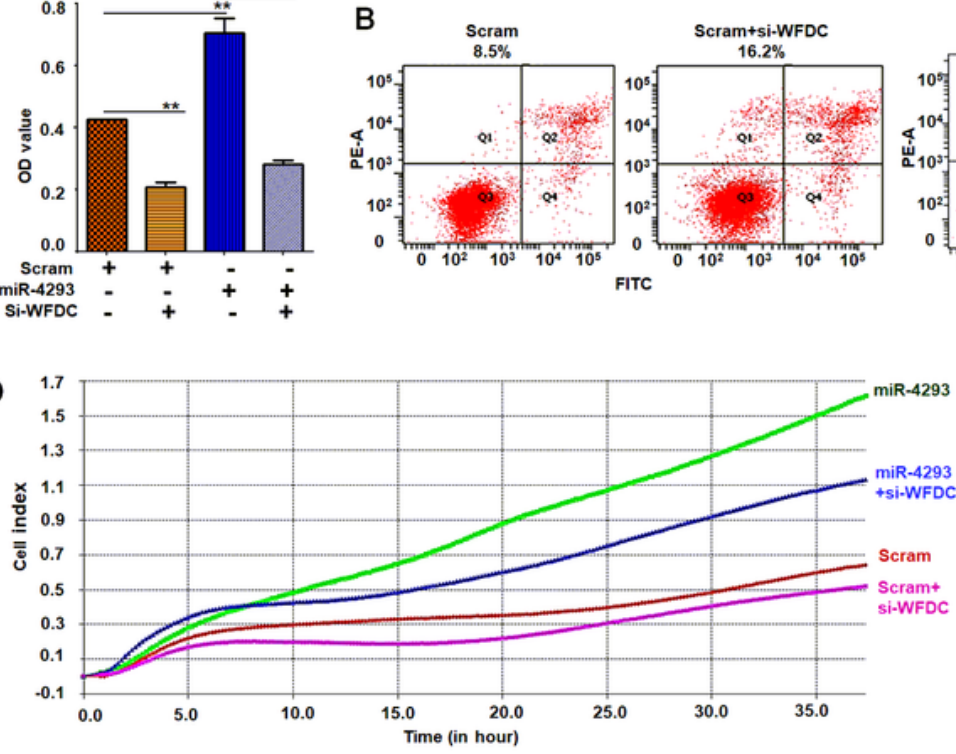
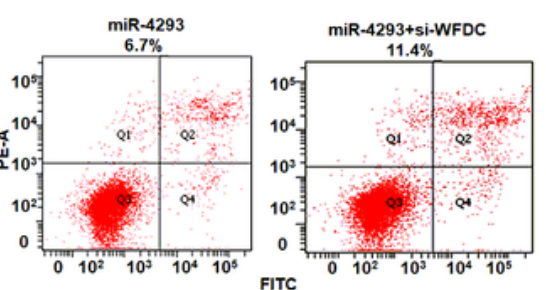

E

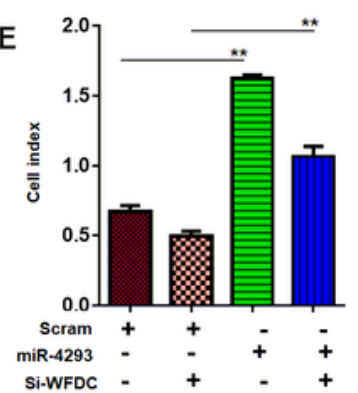

C

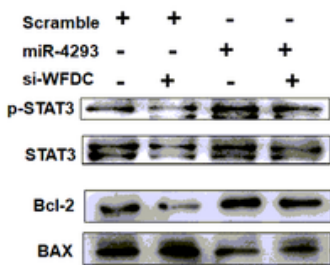

total

caspas

Cleaved-
caspase

GAPDH

$\longrightarrow$

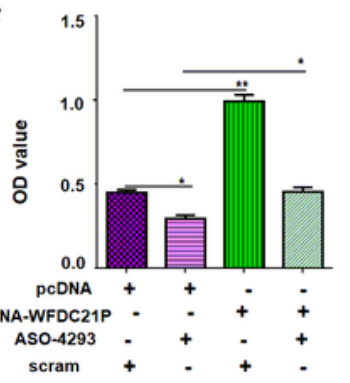

I

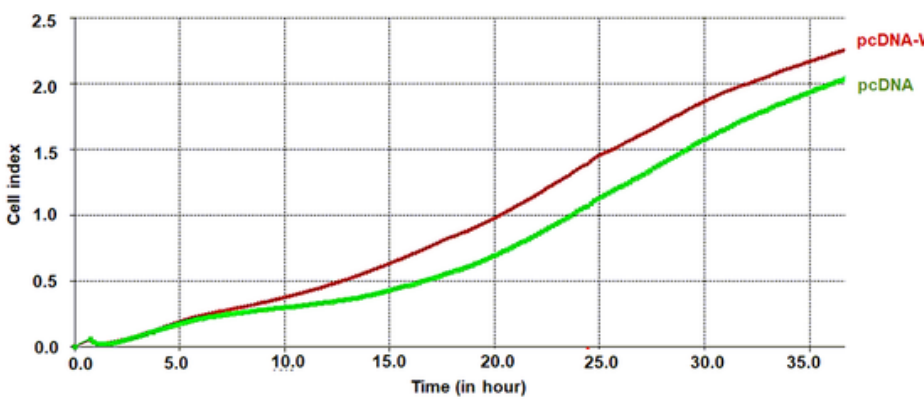

$\mathrm{K}$

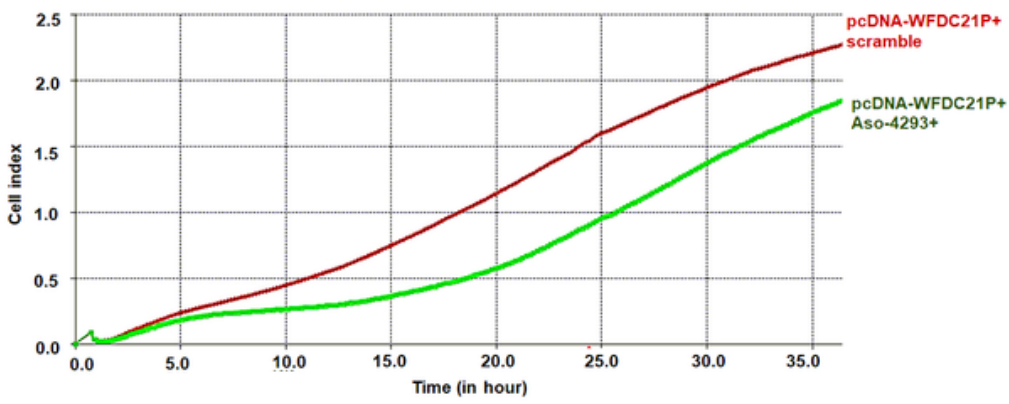

G

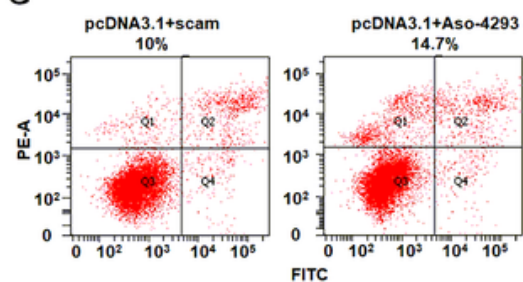

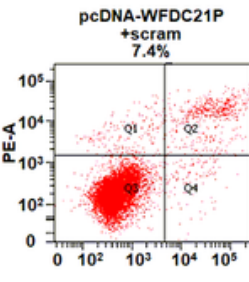
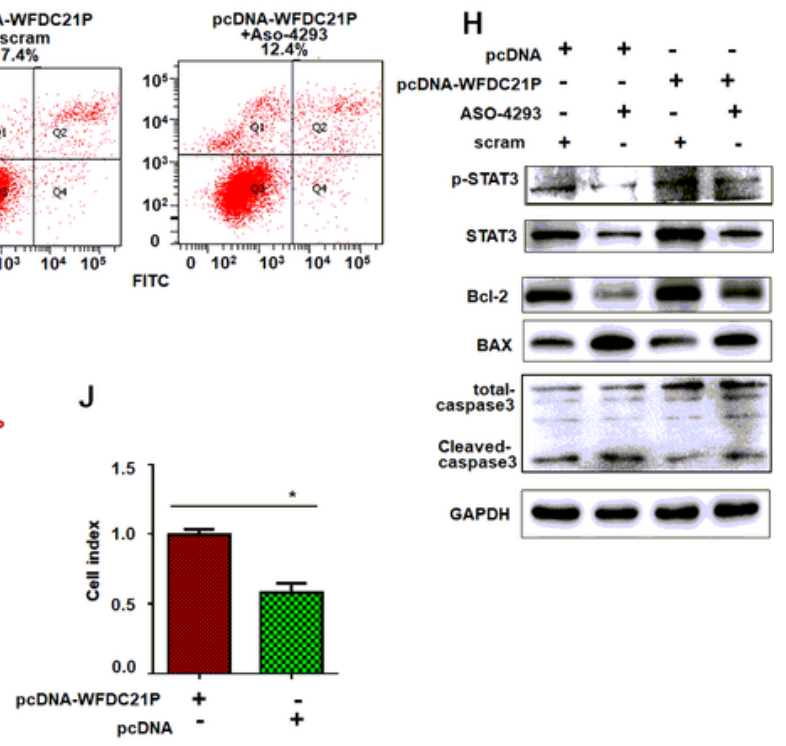

L

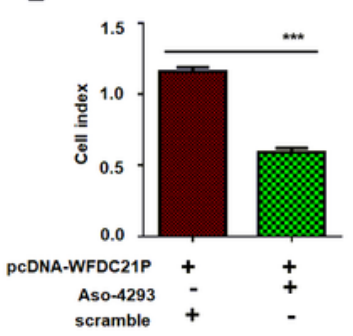

\section{Figure 6}

Knockdown of WFDC21P Attenuates the Oncogenic Role of miR-4293. (A) MTT assays showed proliferation of A549 at $24 \mathrm{~h}$ post-transfection of si-WFDC+scramble, miR-4293, miR-4293+si-WFDC and scramble. Data are expressed as mean \pm SD for triplicate experiments. ${ }^{\star \star} p<0.01$; ANOVA test. (B) FACS analysis showed A549 cell apoptosis at $24 \mathrm{~h}$ post-transfection of si-WFDC+scramble, miR-4293, miR4293+si-WFDC and scramble. (C) Western blot showed level of p-STAT3, Bcl-2, BAX, cleaved-caspase 3, 8, 
and 9 in si-WFDC+scramble-, miR-4293-, miR-4293+si-WFDC- or scramble-treated cells. (D-E) RTCA station analysis showed the migration for A549 with transcription of si-WFDC+ scramble, miR-4293, miR$4293+$ si-WFDC or scramble. (F) MTT assay showed proliferation of A549 at $24 \mathrm{~h}$ post-transfection of pcDNA+ASO-4293, pcDNA-WFDC21P+ scramble, pcDNA-WFDC21P+ ASO-4293 and pcDNA+ scramble. Data are expressed as mean $\pm S D$ for triplicate experiments. ( $P<0.05)$. (G) FACS analysis showed A549 cell apoptosis at $24 \mathrm{~h}$ post-transfection of pcDNA+ASO-4293, pcDNA-WFDC21P+ scramble, pcDNAWFDC21P+ ASO-4293 and pcDNA+ scramble. $(\mathrm{H})$ Western blot showed level of STAT3 phosphorylation and apoptosis-related factors (Bcl-2, Bax and Caspase 3 ) in pcDNA+ASO-4293, pcDNA-WFDC21P+ scramble, pcDNA-WFDC21P+ ASO-4293 and pcDNA+ scramble-treated A549 cells. (I-L) RTCA station analysis of migration for A549. (I) and (J), pcDNA-WFDC21P vs pc-DNA; (K) and (L), pcDNAWFDC21P+scramble vs pcDNA-WFDC21P+ASO-4293. Data are expressed as mean \pm SD for triplicate experiments. ${ }^{*} p<0.05, * \star * p<0.001$; Student's t test.
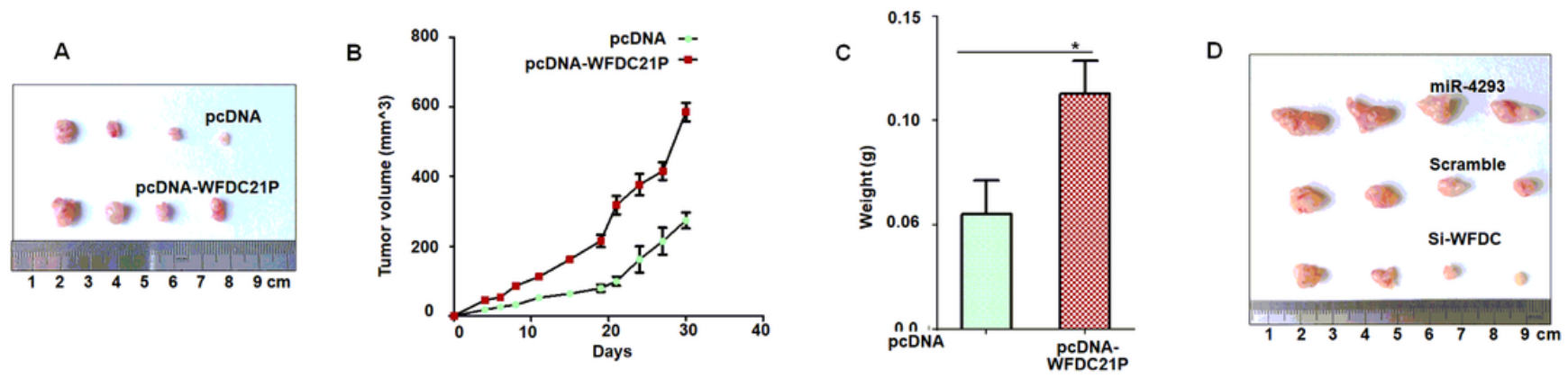

E
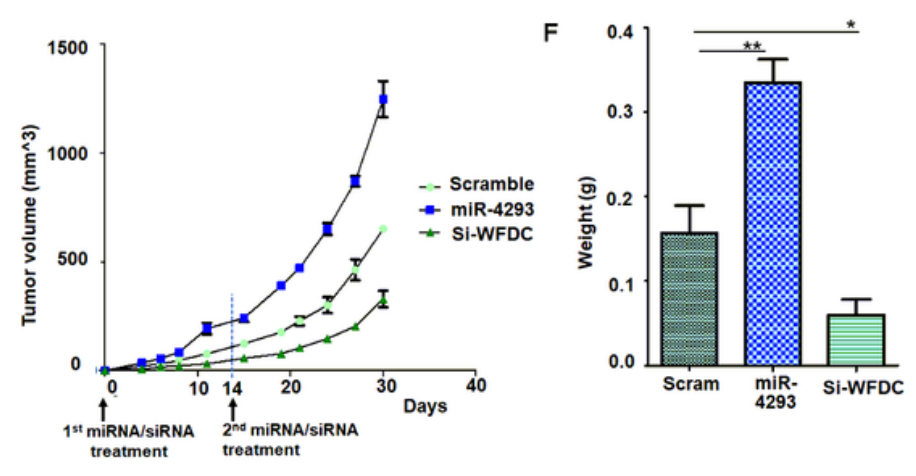

G

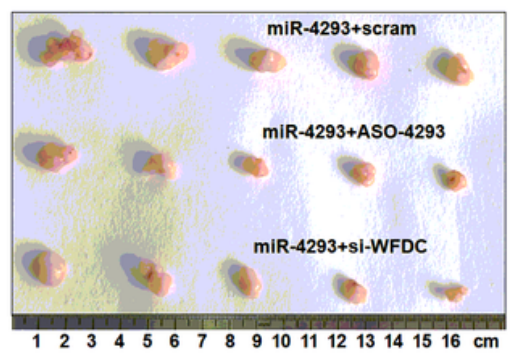

H
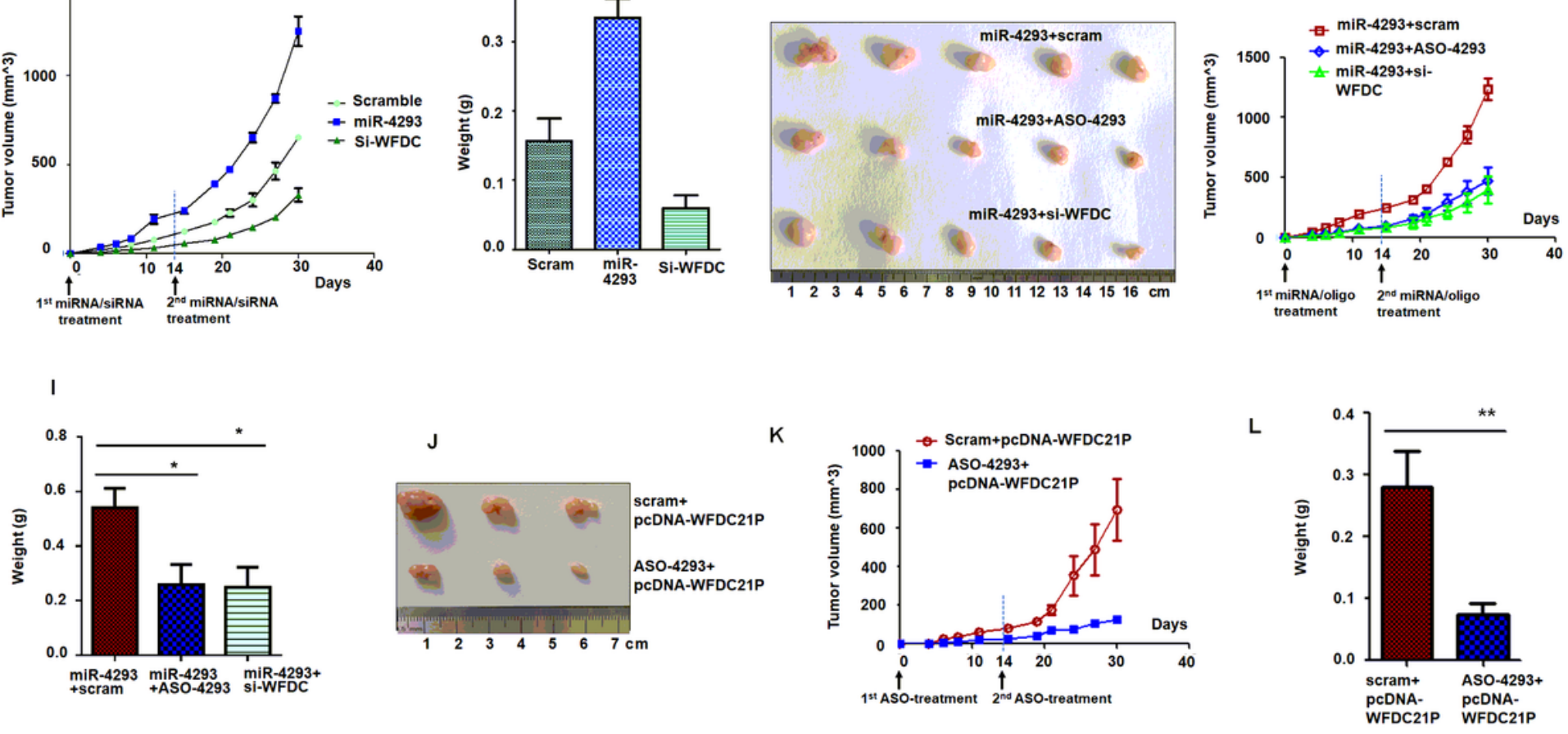

Figure 7

WFDC21P and MiR-4293 Promote Tumorigenesis in vivo. (A-C) Analysis of xenograft of A549 cells treated with pc-DNA or pcDNA-WFDC21P $(n=4)$. Quantitative analysis of tumor volume $(B)$ and tumor 
weight (C) of xenografts. Data are expressed as mean \pm SD. ${ }^{*}<<0.05$; Student's $t$ test. (D-F) Analysis of xenograft tumors of miR-4293-, si-WFDC- and scramble-treated A549 cells ( $n=4)$. Quantitative analysis of tumor volume (E) and tumor weight $(F)$ of xenografts. Data are expressed as mean $\pm S D$. $* * p<0.01$, * $\mathrm{p}<0.05$; ANOVA test. (G-I) Analysis of xenograft tumors of miR-4293-, miR-4293+ASO-4293- and miR4293+WFDC-treated A549 cells ( $n=5)$. Quantitative analysis of tumor volume $(H)$ and tumor weight $(I)$ of xenografts. Data are expressed as mean \pm SD. $* * p<0.01, * p<0.05$; ANOVA test. (J-L) Analysis of xenograft tumors of A549 cells treated with pcDNA-WFDC21P+ ASO-4293 or pcDNA-WFDC21P+scramble $(n=3)$. Quantitative analysis of tumor volume $(K)$ and tumor weight $(L)$ of xenografts. Data are expressed as mean $\pm S D$. ** $p<0.01$; Student's t test.

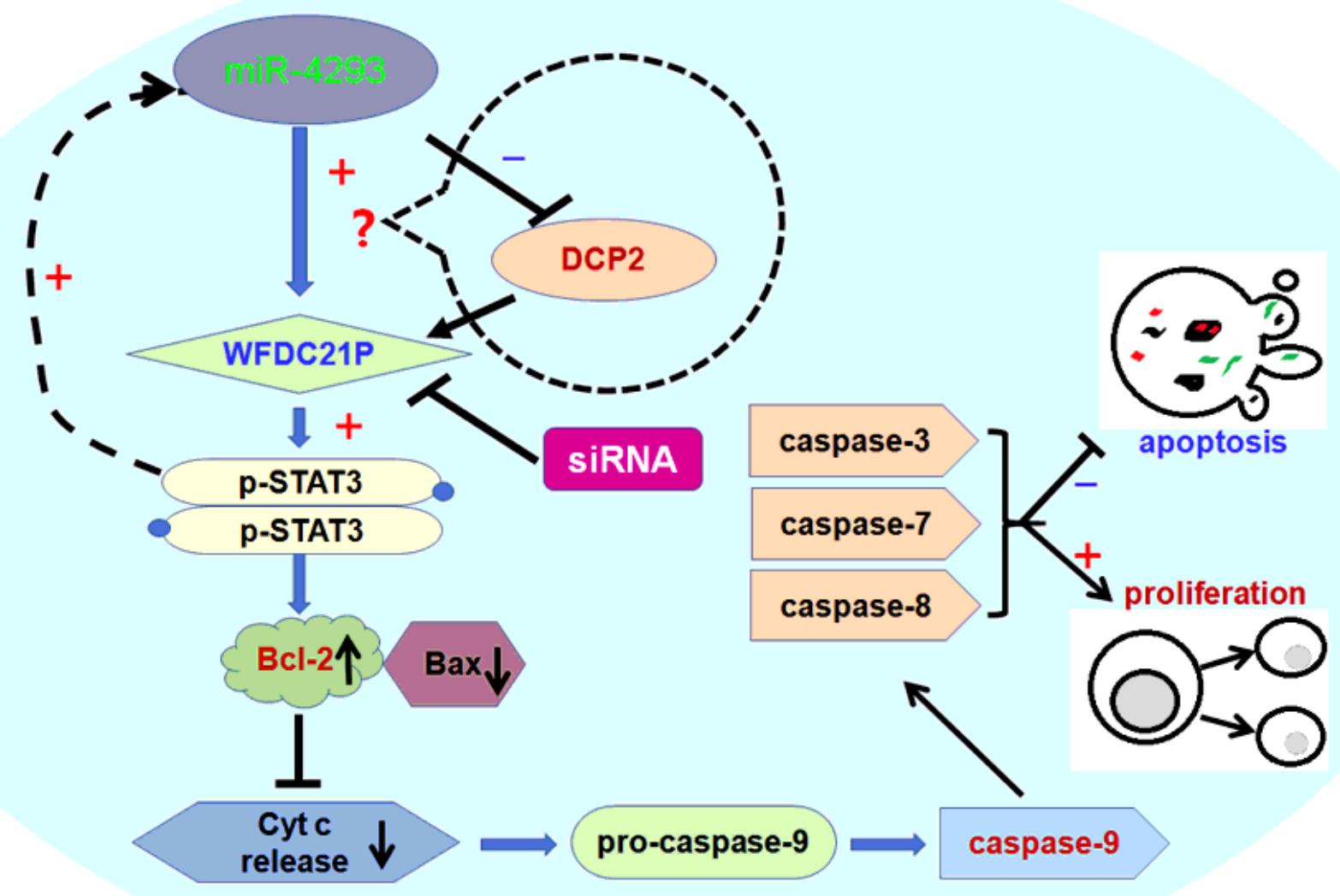

Figure 8

Summary of WFDC21P regulated by miR-4293 through DCP-2

\section{Supplementary Files}

This is a list of supplementary files associated with this preprint. Click to download.

- Supplementaldata.docx

- Supplementaldata.docx 
- Supplementaldata.docx

Page 29/29 